\title{
ON CAUCHY-RIEMANN CIRCLE BUNDLES
}

\author{
Donato Antonio Ciampa
}

\begin{abstract}
Building on ideas of R. Mizner, [17]-[18], and C. Laurent-Thiébaut, [14], we study the CR geometry of real orientable hypersurfaces of a Sasakian manifold. These are shown to be $\mathrm{CR}$ manifolds of $\mathrm{CR}$ codimension two and to possess a canonical connection $D$ (parallelizing the maximally complex distribution) similar to the TanakaWebster connection (cf. [21]) in pseudohermitian geometry. Examples arise as circle subbundles $S^{1} \rightarrow N \stackrel{\pi}{\rightarrow} M$, of the Hopf fibration, over a real hypersurface $M$ in the complex projective space. Exploiting the relationship between the second fundamental forms of the immersions $N \rightarrow S^{2 n+1}$ and $M \rightarrow \mathbf{C P}^{n}$ and a horizontal lifting technique we prove a $\mathrm{CR}$ extension theorem for CR functions on $N$. Under suitable assumptions $\left[\operatorname{Ric}_{D}(Z, \bar{Z})+2 g(Z,(I-a) \bar{Z}) \geq 0, Z \in T_{1,0}(N)\right.$, where $a$ is the Weingarten operator of the immersion $N \rightarrow S^{2 n+1}$ ] on the Ricci curvature $\operatorname{Ric}_{D}$ of $D$, we show that the first Kohn-Rossi cohomology group of $M$ vanishes. We show that whenever $\operatorname{Ric}_{D}(Z, \bar{W})-$ $2 g(Z, \bar{W})=(\mu \circ \pi) g(Z, \bar{W})$ for some $\mu \in C^{\infty}(M), M$ is a pseudo-Einstein manifold.
\end{abstract}

\section{Introduction and statement of main results}

Let $M$ be a differentiable manifold, of class $C^{\infty}$ and real dimension $2 n+k$. A subbundle $T_{1,0}(M)$, of the complexified tangent bundle $T(M) \otimes \mathbf{C}$, of complex rank $n$, is said to be a $C R$ structure on $M$ if

$$
\begin{gathered}
T_{1,0}(M)_{x} \cap T_{0,1}(M)_{x}=(0), \quad x \in M, \\
Z, W \in \Gamma^{\infty}\left(T_{1,0}(M)\right) \Rightarrow[Z, W] \in \Gamma^{\infty}\left(T_{1,0}(M)\right) .
\end{gathered}
$$

The pair $(n, k)$ is the type of the CR structure. A pair $\left(M, T_{1,0}(M)\right)$ is a $C R$ manifold (of type $(n, k)$ ). We set $T_{0,1}(M)=\overline{T_{1,0}(M)}$, where an overbar denotes complex conjugation. If $E \rightarrow M$ is a vector bundle over $M$ then $E_{x}$ denotes the fibre over $x \in M$ and $\Gamma^{\ell}(E)$ denotes the space of sections of class $C^{\ell}$, $\ell \in \mathbf{N} \cup\{\infty, \omega\}$ (eventually defined on an open subset $U \subseteq M$, to be understood from the context). The integer $n$ (respectively $k$ ) is the $C R$ dimension (respectively the $C R$ codimension) of the $\mathrm{CR}$ manifold $\left(M, T_{1,0}(M)\right)$. A CR manifold of type $(n, 1)$, i.e. of CR codimension $k=1$, is said to be of hypersurface type. The terminology is motivated by the fact that every real hypersurface $M$ in $\mathbf{C}^{n+1}$ carries a naturally induced $\mathrm{CR}$ structure $T_{1,0}(M)$ (of type $(n, 1)$ ) given by

Received June 7, 2004; revised October 18, 2004. 
$T_{1,0}(M)_{x}=\left[T_{x}(M) \otimes_{\mathbf{R}} \mathbf{C}\right] \cap T^{1,0}\left(\mathbf{C}^{n+1}\right)_{x}, x \in M$, where $T^{1,0}\left(\mathbf{C}^{n+1}\right)$ is the holomorphic tangent bundle, i.e. if $z^{1}, \ldots, z^{n+1}$ are complex coordinates on $\mathbf{C}^{n+1}$, then $T^{1,0}\left(\mathbf{C}^{n+1}\right)$ is the span of $\left\{\partial / \partial z^{j}: 1 \leq j \leq n+1\right\}$. Such CR manifolds are referred to as embedded.

An important class of CR manifolds (of arbitrary type $(n, k)$ ) consists of the proper CR submanifolds, in the sense of A. Bejancu, [3]. Given a Hermitian manifold $\left(M_{0}, J, g_{0}\right)$, with the complex structure $J$ and the Hermitian metric $g_{0}$, a pair $(M, \mathscr{D})$ consisting of a submanifold $M$ of $M_{0}$ and of a smooth distribution, $\mathscr{D}: x \in M \mapsto \mathscr{D}_{x} \subseteq T_{x}(M)$, is a $C R$ submanifold if i) $\mathscr{D}$ is $J$-invariant, i.e. $J_{x}\left(\mathscr{D}_{x}\right)=\mathscr{D}_{x}, x \in M$, and ii) the orthogonal complement $\mathscr{D}^{\perp}$ (with respect to $\left.g:=j^{*} g_{0}, j: M \subset M_{0}\right)$ of $\mathscr{D}$ in $T(M)$ is $J$-anti-invariant, i.e. $J_{x}\left(\mathscr{D}_{x}^{\perp}\right) \subseteq T_{x}(M)^{\perp}$, $x \in M$. Here $\mathscr{V}(j)=T(M)^{\perp}$ is the normal bundle (of the given immersion $j$ : $\left.M \subset M_{0}\right)$.

Let $(M, \mathscr{D})$ be a CR submanifold of the Hermitian manifold $\left(M_{0}, J, g_{0}\right)$. Set $n:=\operatorname{dim}_{\mathbf{C}} \mathscr{D}_{x}, k:=\operatorname{dim}_{\mathbf{R}} \mathscr{D}_{x}^{\perp}$, for any $x \in M$ (so that $\operatorname{dim} M=2 n+k$ ). A $\mathrm{CR}$ submanifold $(M, \mathscr{D})$ is generic if $k$ and the codimension of $M$ in $M_{0}$ coincide. A CR submanifold $(M, \mathscr{D})$ is said to be proper if both the holomorphic and totally real distributions are nonzero $(n \neq 0$ and $k \neq 0)$.

By a result in [5] (referred to as the Blair-Chen theorem, in the sequel), every proper CR submanifold $(M, \mathscr{D})$ of a Hermitian manifold is a CR manifold. Indeed, let $J_{M}$ be the restriction of $J$ to $\mathscr{D}$. Moreover, let $J_{M}^{\mathrm{C}}$ be the extension (by complex linearity) of $J_{M}$ to $\mathscr{D} \otimes \mathbf{C}$ and set $T_{1,0}(M):=\operatorname{Eigen}\left(J_{M}^{\mathbf{C}}\right.$, i), i.e. $T_{1,0}(M)_{x}$ is the eigenspace of $\left(J_{M}^{\mathbf{C}}\right)_{x}$ corresponding to the eigenvalue $i=\sqrt{-1}$, $x \in M$. Then, by the previously quoted result (cf. [5], or Theorem 2.1 in [29], p. 83), $\left(M, T_{1,0}(M)\right)$ is a CR manifold of type $(n, k)$.

A CR structure on a manifold is a reformulation (in terms of fibre bundles) of the tangential Cauchy-Riemann equations induced (on a real submanifold) by the Cauchy-Riemann equations (on the ambient complex manifold). If $\Omega=\{\rho>0\} \subset \mathbf{C}^{n+1}$ is a smooth domain, then $M:=\partial \Omega$ inherits a $\mathrm{CR}$ structure $T^{1,0}(M)$ (as a real hypersurface in $\mathbf{C}^{n+1}$ ) locally spanned by $L_{\alpha}=$ $\sum_{j=1}^{n+1} a_{\alpha}^{j}(z) \partial / \partial z^{j}, 1 \leq \alpha \leq n$, where $\sum_{j=1}^{n+1} a_{\alpha}^{j}(z) \partial \rho / \partial z^{j}=0,1 \leq \alpha \leq n$ (i.e. the $L_{\alpha}$ 's are purely tangential first order differential operators). The restriction $u$ to $M$ of a holomorphic function $f$ on an open neighborhood of $M$ satisfies the tangential Cauchy-Riemann equations

$$
L_{\bar{\alpha}} u \equiv \sum_{j=1}^{n+1} \overline{a_{\alpha}^{j}(z)} \frac{\partial u}{\partial \bar{z}^{j}}=0, \quad 1 \leq \alpha \leq n .
$$

If $\left(M, T_{1,0}(M)\right)$ is an arbitrary CR manifold (of arbitrary, but fixed, type) let us consider the differential operator

$$
\bar{\partial}_{M}: C^{\infty}(M, \mathbf{C}) \rightarrow \Gamma^{\infty}\left(T_{0,1}(M)^{*}\right)
$$

given by $\left(\bar{\partial}_{M} u\right) \bar{Z}:=\bar{Z}(u)$, for any $Z \in \Gamma^{\infty}\left(T_{1,0}(M)\right), \quad u \in C^{\infty}(M, \mathbf{C})$. The equations

$$
\bar{\partial}_{M} u=0
$$


are also referred to as the tangential Cauchy-Riemann equations (although not any longer confined to the CR codimension 1 case) and coincide locally with (1.3) when $M=\partial \Omega$.

When $M$ is a real submanifold of a complex manifold $V$ (endowed with the induced CR structure), a fundamental problem of complex analysis in several variables is whether, given a solution $u$ to (1.4), there is a holomorphic function extending $u$ (e.g. in a neighborhood of $M$ ). The solutions to the given problem (perhaps under additional assumptions on the geometry of $M$, e.g. imposed to its Levi form) occupy a large space in the specialized literature (cf. e.g. A. Boggess, [6], p. 189-260).

The holomorphic extension problem may be solved in the real analytic case by a result of G. Tomassini, [22]. A CR manifold is real analytic if $M$ is a $C^{\omega}$ manifold and its $\mathrm{CR}$ structure is locally spanned by real analytic sections. Let $M \subset \mathbf{C}^{n}$ be a real analytic embedded CR manifold. Let $u: M \rightarrow \mathbf{C}$ be a real analytic $C R$ function, i.e. a $C^{\omega}$ solution to (1.4). Then, by the Tomassini theorem (cf. op. cit.), there is a neighborhood $U$ of $M$ in $\mathbf{C}^{n}$ and a unique holomorphic function $f: U \rightarrow \mathbf{C}$ whose trace on $M$ is $u$.

Let $N_{0}$ be a manifold carrying an almost contact metric (a.ct.m.) structure $\left(\phi, \xi, \eta_{0}, g_{0}\right)$. A pair $(N, \mathscr{D})$ consisting of a submanifold $N$ of $N_{0}$ and a smooth distribution $\mathscr{D}: x \in N \mapsto \mathscr{D}_{x} \subseteq T_{x}(N)$ is said to be a contact $C R$ submanifold of $N_{0}$ if i) $\mathscr{D}$ is $\phi$-invariant, i.e. $\phi_{x}\left(\mathscr{D}_{x}\right) \subseteq \mathscr{D}_{x}, x \in N$, and ii) the orthogonal complement $\mathscr{D}^{\perp}$ of $\mathscr{D}$ (with respect to $g:=i^{*} g_{0}$ ) in $T(N)$ is $\phi$-anti-invariant, i.e. $\phi_{x}\left(\mathscr{D}_{x}^{\perp}\right) \subseteq T_{x}(N)^{\perp}, x \in N$. Here $\mathscr{V}(i)=T(N)^{\perp}$ is the normal bundle of the immersion $i: N \subset N_{0}$.

Let $(N, \mathscr{D})$ be a contact $\mathrm{CR}$ submanifold of the a.ct.m. manifold $N_{0}$. $(N, \mathscr{D})$ is generic if $\operatorname{dim}_{\mathbf{R}} \mathscr{D} \perp x=\operatorname{codim}(N), x \in N . \quad(N, \mathscr{D})$ is proper if $\mathscr{D} \neq(0)$ and $\mathscr{D}^{\perp} \neq(0)$.

The concept of a contact CR submanifold (with the additional assumption that $\xi$, the contact field of the ambient manifold $N_{0}$, is tangent to $N$ ) is due to K. Yano \& M. Kon, [29], p. 48. Taking into consideration the Blair-Chen theorem, it is natural to ask whether a contact CR submanifold is a CR manifold (and of what type). Let $(N, \mathscr{D})$ be a contact $\mathrm{CR}$ submanifold of the Sasakian manifold $N_{0}$, tangent to the contact field $\xi$ of $N_{0}$. A simple argument shows that either $\xi \in \mathscr{D}$ or $\xi \in \mathscr{D}^{\perp}$. However, to avoid the disjunction of cases, let $H(N)$ be the orthogonal complement of $\mathbf{R} l \xi$ in $\mathscr{D}$ [where $l: T(N) \rightarrow \mathscr{D}$ is the canonical projection], i.e. $\mathscr{D}=H(N) \oplus \mathbf{R} l \xi$. Also, let $H(N)^{\perp}$ be the orthogonal complement of $H(N)$ in $T(N)$. $H(N)$ is $\phi$-invariant, $H(N)^{\perp}$ is $\phi$-anti-invariant, hence $(N, H(N))$ is a contact $\mathrm{CR}$ submanifold. Moreover $\xi \in H(N)^{\perp}$ and the restriction $J$ of $\phi$ to $H(N)$ is a complex structure on $H(N)$. We establish

THEOREM 1. $T_{1,0}(N):=[T(N) \otimes \mathbf{C}] \cap T_{1,0}\left(N_{0}\right)$ is a CR structure on $N$, of type $(n, k)$, where $n:=\operatorname{dim}_{\mathbf{C}} H(N)_{x}$ and $k:=\operatorname{dim}_{\mathbf{R}} H(N)_{x}^{\perp}, x \in N$. Therefore $\left(N, T_{1,0}(N)\right)$ is a $C R$ manifold and $i: N \subset N_{0}$ is a CR immersion.

By a result in [13] (referred to as the Ianus theorem, in the sequel), every Sasakian manifold $N_{0}$ admits a natural CR structure $T_{1,0}\left(N_{0}\right):=\{X-i \phi X$ : 
$\left.X \in \operatorname{Ker}\left(\eta_{0}\right)\right\} . \quad$ A $C R$ immersion is a $C^{\infty}$ map $f: N \rightarrow N^{\prime}$ of CR manifolds such that $f$ is an immersion and a $C R$ map, i.e. $\left(d_{x} f\right) T_{1,0}(N)_{x} \subseteq T_{1,0}\left(N^{\prime}\right)_{f(x)}, x \in N$. For simplicity Theorem 1 is stated for CR submanifolds of Sasakian manifolds (while the Ianus theorem only requires that $\left(\phi, \xi, \eta_{0}\right)$ is normal).

An important class of contact CR submanifolds of an odd-dimensional sphere consists of the total spaces of the (circle) subbundles of the Hopf fibration, over a CR submanifold of the complex projective space. Precisely, let $M$ be a CR submanifold in $\mathbf{C P}^{n}$ and let $N:=\pi_{0}^{-1}(M)$, where $\pi_{0}: S^{2 n+1} \rightarrow \mathbf{C P}^{n}$ is the projection. Then $N$ is a contact $\mathrm{CR}$ submanifold of $S^{2 n+1}$ (carrying the standard Sasakian structure) and $S^{1} \rightarrow N \stackrel{\pi}{\rightarrow} M$ is a principal subbundle of the Hopf fibration $S^{1} \rightarrow S^{2 n+1} \rightarrow \mathbf{C P}^{n}$, where $\pi:=\left.\pi_{0}\right|_{N} . \quad S^{1} \rightarrow N \stackrel{\pi}{\rightarrow} M$ is said to be a Cauchy-Riemann circle bundle and is the central notion of this paper. Indeed, when the given contact CR submanifold $N$ is (the total space of) a $S^{1}$-bundle over a CR submanifold $M \subset \mathbf{C P}^{n}$, the pullback $\eta:=i^{*} \eta_{0}$ of the contact form of $S^{2 n+1}$ is a connection form in $S^{1} \rightarrow N \rightarrow M$, a fact which enables one to consider horizontal lifts of geometric objects on $M$ (e.g. the first and the second fundamental forms of $j: M \subset \mathbf{C P}^{n}$, the induced and normal connections, the Weingarten operator, etc.). This leads to a precise description of the geometry of (the second fundamental form of) $j: M \subset \mathbf{C P}^{n}$ in terms of the geometry of (the second fundamental form of ) $i: N \subset S^{2 n+1}$ and successive applications. We have

TheOrem 2. Let $n, k \in \mathbf{Z}, n, k>0$, and let $S^{1} \rightarrow N \rightarrow M$ be a CauchyRiemann circle bundle over a real analytic compact connected $C R$ submanifold $M \subset \mathbf{C P}^{n+k-1}$, with $\operatorname{dim} N=2 n+k$ and $\operatorname{dim}_{\mathbf{R}} H(N)_{x}=2 n, x \in N$. If $N$ is generic and the contact vector field of $S^{2(n+k)-1}$ lies in the invariant distribution of $N$, then there is a saturated subset $Q \subset S^{2(n+k)-1}$ such that $Q \supset N$, and there is a subbundle $S^{1} \rightarrow N^{\prime} \rightarrow M^{\prime}$ of the Hopf fibration $S^{1} \rightarrow S^{2(n+k)-1} \rightarrow \mathbf{C P}^{n+k-1}$, over a submanifold $M^{\prime} \subset \mathbf{C P}^{n+k-1}$ of real dimension $2 n+k$, such that $Q \supset N^{\prime}$ and, for every basic $C R$ function $u \in C R^{\omega}(N)$ there is an open and saturated set $U \subseteq S^{2(n+k)-1}$ such that $U \supset Q$ and there is a basic $C R$ function $f \in C R^{\omega}(U)$ which extends $u$, i.e. $\left.f\right|_{N}=u$.

Theorem 2 is an immediate corollary of the Tomassini theorem and of a result by $\mathrm{R}$. O. Wells (cf. [25]) according to which, given a compact and connected submanifold $M \subset V$ in a complex manifold $V$, if $M$ is a generic CR manifold of type $(n, k)$, with $n>0$, the holomorphic hull of $M$ contains a manifold $M^{\prime}$ of higher dimension (i.e. $\operatorname{dim} M^{\prime}=2 n+k+1$ ). While Theorem 2 is a rather elementary consequence of the above mentioned results (of G. Tomassini and R. O. Wells, cf. op. cit.), it leads naturally to the question: which real submanifolds of a Sasakian manifold are generic? The following result establishes a necessary topological condition.

THEOREM 3. Let $(N, \mathscr{D})$ be a contact $C R$ submanifold of a Sasakian manifold $N_{0}$. Let us assume that $N$ is generic and that the tangential component of the 
contact vector $\xi_{0}$ of $N_{0}$ lies in the invariant distribution [i.e. $\left.\xi:=\tan \left(\xi_{0}\right) \in \mathscr{D}\right]$. Then the Euler-Poincaré characteristic of $N$ vanishes [i.e. $\chi(N)=0]$.

Theorem 3 is a contact analogue of a result by R. O. Wells (cf. theorem 1 in [26], p. 124). If $M \subset \mathbf{C P}^{n}$ is a compact CR submanifold and $N:=\pi_{0}^{-1}(M) \subset$ $S^{2 n+1}$, then it is immediate that $\chi(N)=0$. Indeed, in this case $N$ is tangent to the contact vector field $\xi$ of $S^{2 n+1}$ (and $\xi(x) \neq 0$ for any $x \in N$ ).

Every nondegenerate $\mathrm{CR}$ manifold $M$, of hypersurface type, on which a contact form $\theta$ is fixed, admits a unique linear connection parallelizing the Levi distribution $\operatorname{Ker}(\theta)$, its complex structure, the Webster metric $g_{\theta}$, and whose torsion is pure. This is the Tanaka-Webster connection of $(M, \theta)$ (discovered independently by N. Tanaka, [21], and S. Webster, [24]). The discovery of the Tanaka-Webster connection revealed itself of a certain importance in understanding pseudohermitian geometry. For instance, the Chern-Moser tensor (which is well known to vanish if and only if $M$ is locally CR equivalent to a sphere) may be expressed in terms of the curvature of the Tanaka-Webster connection. Similarly, the Fefferman metric (cf. C. Fefferman, [11]) may be expressed in terms of the connection forms and scalar curvature of the Tanaka-Webster connection (cf. J. M. Lee, [15]). These and other issues (for instance those tied to the geometry of Yang-Mills fields on CR manifolds, cf. H. Urakawa, [23], or to the geometry of CR immersions, cf. S. Dragomir, [8], etc.) lead to the fundamental problem of building an analogue of the Tanaka-Webster connection in higher CR codimension $(k \geq 2)$. Indeed this is the case of real hypersurfaces $N \subset S^{2 n+1}$ (any such $N$ has CR codimension 2).

The first attempt to solve the problem belongs to R. Mizner (cf. [18]), who built a connection $\nabla$ generalizing the Tanaka-Webster connection to the case of a CR manifold $M$ of $\mathrm{CR}$ codimension $\geq 2$ under the assumption that $M$ is nondegenerate and the Levi distribution $H(M)$ admits a complement $E$ which is trivial as a bundle over $M$. Since (as in the CR codimension one case) one of the requirements for building $\nabla$ is that the Levi form be parallel, the nondegeneracy assumption is quite natural. As to the triviality of $E$, while it follows from the mere orientability of $M$ in the CR codimension one case, it is not $a$ priori clear what obstructions there are towards of $E \approx M \times \mathbf{R}^{k}$, when $k \geq 2$ (neither does R. Mizner investigate such obstructions).

In this paper we adopt an alternative to R. Mizner's ideas, that is we request that the connection parallelizes the induced metric (rather than the Levi form $\mathscr{L}$ ) and we replace, here as well as in the classical case, the symmetry assumption by a suitable "purity" axiom. Precisely, let $N$ be a real orientable hypersurface in a Sasakian manifold $\left(N_{0},\left(\phi, \xi, \eta_{0}, g_{0}\right)\right)$ (for instance $\left.N_{0}=S^{2 n+1}\right)$, tangent to the contact vector field of $N_{0}$. Let $v$ be a global unit normal vector field on $N$, and set $U:=\phi v$. Then $U$ is tangential (i.e. $U \in T(N)$ ) and orthogonal to $\xi$. Let $H(N)$ be the orthogonal complement of $\mathbf{R} U \oplus \mathbf{R} \xi$ in $T(N)$. We establish

THeORem 4. There is a unique linear connection $D$ on $N$ satisfying the following properties 
1) $H(N)$ is parallel with respect to $D$,

2) $D g=0$, where $g=i^{*} g_{0}, i: N \subset N_{0}$,

3) $D J=0$, where $J=\left.\phi\right|_{H(N)}$,

4) the torsion $T_{D}$ of $D$ is pure, i.e.

$$
\begin{gathered}
T_{D}(Z, W)=0, \quad T_{D}(Z, \bar{W})=-2 i \mathscr{L}(Z, \bar{W}), \quad Z, W \in T_{1,0}(N), \\
\tau \circ J+J \circ \tau=0, \quad \rho \circ J+J \circ \rho=0,
\end{gathered}
$$

where $\tau X:=T_{D}(\xi, X), \rho X:=T_{D}(U, X), X \in T(N)$, and

5) $D \xi=0, D U=0$.

In analogy with pseudohermitian geometry and the fundamental role played there by the Tanaka-Webster connection, we expect several applications (in the study of CR structures of real orientable hypersurfaces $N \subset S^{2 n+1}$ ) of the canonical connection furnished by Theorem 4. For instance, when $N$ fibres in circles over a real hypersurface in $\mathbf{C P}^{n}$, we establish the following "vanishing theorem"

TheOREM 5. Let $S^{1} \rightarrow N \rightarrow M$ be a Cauchy-Riemann circle bundle over a compact orientable real hypersurface $M \subset \mathbf{C P}^{n}$. Assume that $M$ satisfies the contact condition and

$$
\operatorname{Ric}_{D}(Z, \bar{Z})+2 g(Z,(\mathrm{I}-a) \bar{Z}) \geq 0, \quad Z \in T_{1,0}(N),
$$

where $\operatorname{Ric}_{D}$ is the Ricci tensor of the canonical connection $D$ on $N$ and $a$ is the Weingarten operator corresponding to a choice of global unit normal $v$ on $N$. Then the first Kohn-Rossi cohomology group of $M$ vanishes, i.e. $H^{0,1}\left(M ; \bar{\partial}_{M}\right)=0$.

Given a nondegenerate $\mathrm{CR}$ manifold $M$ of $\mathrm{CR}$ dimension $n$, a contact form $\theta$ on $M$ is pseudo-Einstein if the pseudohermitian Ricci tensor (of the Tanaka-Webster connection of $(M, \theta))$ is proportional to the Levi form, i.e. $R_{\alpha \bar{\beta}}=\mu h_{\alpha \bar{\beta}}$ for some $\mu \in C^{\infty}(M)$ (a posteriori $\mu=(1 / n) \rho$, where $\rho=h^{\alpha \bar{\beta}} R_{\alpha \bar{\beta}}$ is the pseudohermitian scalar curvature of $(M, \theta))$. Another application of Theorem 4 is

TheORem 6. Let $S^{1} \rightarrow N \stackrel{\pi}{\rightarrow} M$ be a Cauchy-Riemann circle bundle over an orientable real hypersurface $M \subset \mathbf{C P}^{n}$ satisfying the contact condition. Then there is $\mu \in C^{\infty}(M)$ such that

$$
\operatorname{Ric}_{D}(Z, \bar{W})-2 g(Z, a \bar{W})=(\mu \circ \pi) g(Z, \bar{W}), \quad Z, W \in T_{1,0}(N),
$$

if and only if $\theta$ is pseudo-Einstein of pseudohermitian scalar curvature $\rho=$ $(n-1)(\mu+2)$. Here $\theta(X)=-G\left(X, J_{\mathrm{CP}^{n} v_{M}}\right), X \in T(M)$. In particular, if (1.6) holds, then the CR structure of $M$ has a vanishing first Chern class.

Acknowledgements. The author wishes to express his gratitude towards professor Sorin Dragomir for his constant guidance during the elaboration of this paper and to the Referee for pointing out certain corrections. 


\section{CR submanifolds}

\subsection{CR submanifolds of Hermitian manifolds}

Let $j: M \subset M_{0}$ be a submanifold of a Hermitian manifold $\left(M_{0}, J, g_{0}\right)$ and $g:=j^{*} g_{0}$ the induced metric. Let $T(M)^{\perp} \rightarrow M$ be the normal bundle of the given immersion. We shall need the Gauss and Weingarten formulae

$$
\begin{aligned}
& \nabla_{X}^{0} Y=\nabla_{X} Y+h(X, Y) \\
& \nabla_{X}^{0} V=-a_{V} X+\nabla_{X}^{\perp} V
\end{aligned}
$$

for any $X, Y \in \mathscr{X}(M)$ and $V \in \Gamma^{\infty}\left(T(M)^{\perp}\right)$. Here $\nabla^{0}$ is the Levi-Civita connection of $\left(M_{0}, g_{0}\right), \nabla$ is the induced connection, $h$ is the second fundamental form of the given immersion, $a_{V}$ is the Weingarten operator (associated to the normal section $V$ ) and $\nabla^{\perp}$ is the normal connection. Let $\tan _{x}$ and nor ${ }_{x}$ be the projections associated to the direct sum decomposition

$$
T_{x}\left(M_{0}\right)=\left[\left(d_{x} j\right) T_{x}(M)\right] \oplus T_{x}(M)^{\perp}, \quad x \in M .
$$

We set

$$
\mathrm{P} X=\tan (J X), \quad \mathrm{F} X=\operatorname{nor}(J X), \quad \mathrm{t} V=\tan (J V), \quad \mathrm{f} V=\operatorname{nor}(J V) .
$$

Then

$$
\mathrm{P}^{2}=-\mathrm{I}-\mathrm{tF}, \quad \mathrm{FP}+\mathrm{fF}=0, \quad \mathrm{f}^{2}=-\mathrm{I}-\mathrm{Ft}, \quad \mathrm{Pt}+\mathrm{tf}=0 .
$$

The identities (2.3) are commonly stated for submanifolds of Kählerian manifolds. However, an inspection of the proof (cf. e.g. [29], p. 77) shows that (2.3) hold when the ambient space is but Hermitian. We may state the following

THEOREM 7. A submanifold $M$ of a Hermitian manifold is a CR submanifold if and only if $\mathrm{FP}=0$. If this is the case then $\mathscr{D}:=\operatorname{Ker}\left(\mathrm{I}+\mathrm{P}^{2}\right)$ is $J$-invariant (while the orthogonal complement of $\mathscr{D}$ in $T(M)$ is $J$-anti-invariant).

Theorem 7 was first proved by K. Yano \& M. Kon, (cf. Theor. 3.2 in [29], p. 87) for submanifolds of Kählerian manifolds, and the proof is a verbatim repetition of their arguments.

\subsection{Contact CR submanifolds of a.ct.m. manifolds}

Let $N$ be a real $(2 n+1)$-dimensional manifold. A synthetic object $(\phi, \xi, \eta)$ consisting of a tensor field $\phi$ of type $(1,1)$, a tangent vector field $\xi$, and a 1 -form $\eta$, is an almost contact structure on $N$ if

$$
\phi^{2}=-\mathrm{I}+\eta \otimes \xi, \quad \eta(\xi)=1, \quad \phi \xi=0 .
$$

An almost contact manifold is a (odd dimensional) manifold endowed with a fixed almost contact structure. Let $(N,(\phi, \xi, \eta))$ be an almost contact manifold. A Riemannian metric $g$ is compatible with $(\phi, \xi, \eta)$ if $g(\phi X, \phi Y)=g(X, Y)-$ $\eta(X) \eta(Y)$. Then $g(X, \xi)=\eta(X)$. An almost contact metric (a.ct.m.) structure 
$(\phi, \xi, \eta, g)$ consists of an almost contact structure $(\phi, \xi, \eta)$ and a compatible metric $g$. Given an a.ct.m. structure $(\phi, \xi, \eta, g)$ we set $\Omega(X, Y):=g(X, \phi Y) . \quad(\phi, \xi, \eta, g)$ is a contact metric structure if $\Omega=d \eta$ (the contact condition). If this is the case $\eta \wedge \Omega^{n}$ is a volume form, hence $\eta$ is a contact form on $N$. Each orientable real hypersurface $N$ of a Hermitian manifold $\left(M_{0}, J, g_{0}\right)$ carries a naturally induced a.ct.m. structure. Indeed, let $v$ be a unit normal field on $N$. Then $\xi:=J v$ is tangent to $N$. Moreover if $g:=j^{*} g_{0}, \phi X:=\tan (J X)$ and $\eta(X):=g(X, \xi)$, for $X \in T(N)$, then $(\phi, \xi, \eta, g)$ is an a.ct.m. structure on $N$. An a.ct.m. structure $(\phi, \xi, \eta)$ on $N$ is normal if $N^{(1)}:=[\phi, \phi]+2(d \eta) \otimes \xi$ vanishes.

A Sasakian structure is a contact metric structure $(\phi, \xi, \eta, g)$ with $N^{(1)}=0$. A Sasakian manifold is an odd dimensional manifold endowed with a fixed Sasakian structure. The underlying Riemannian metric is usually referred to as a Sasakian metric. Sasakian structures are characterized among a.ct.m. structures by

$$
\left(\nabla_{X} \phi\right) Y=g(X, Y) \xi-\eta(Y) X,
$$

(cf. D. E. Blair, [4], p. 73). Here $\nabla$ is the Levi-Civita connection of $(N, g)$. By the Ianuş theorem (cf. S. Ianuş, [13], or D. E. Blair, [4], p. 61) for each almost contact normal structure $(\phi, \xi, \eta)$ there is a naturally associated CR structure. Indeed $T_{1,0}(N):=\{X-i \phi X: X \in \operatorname{Ker}(\eta)\}$ is an almost CR structure. Moreover, normality $\left(N^{(1)}=0\right)$ implies formal integrability.

Let $(N, \mathscr{D})$ be a contact $\mathrm{CR}$ submanifold of an a.ct.m. manifold $\left(N_{0}\right.$, $\left.\left(\phi, \xi, \eta_{0}, g_{0}\right)\right)$, tangent to the contact vector $(\xi \in T(N))$. Let $l$ and $l^{\perp}$ be the projections associated to the direct sum decomposition $T(N)=\mathscr{D} \oplus \mathscr{D}^{\perp}$. Next, for any $X \in T(N)$ and $V \in T(N)^{\perp}$ we set

$$
\mathrm{P} X=\tan (\phi X), \quad \mathrm{F} X=\operatorname{nor}(\phi X), \quad \mathrm{t} V=\tan (\phi V), \quad \mathrm{f} V=\operatorname{nor}(\phi V) .
$$

Then

$$
\mathrm{P}^{2}=-\mathrm{I}-\mathrm{tF}+\eta \otimes \xi, \quad \mathrm{FP}+\mathrm{fF}=0, \quad \mathrm{f}^{2}=-\mathrm{I}-\mathrm{Ft}, \quad \mathrm{Pt}+\mathrm{tf}=0 .
$$

If $g$ is the induced metric $\left(g=i^{*} g_{0}, i: N \subset N_{0}\right)$, then

$$
g_{0}(\mathrm{~F} X, V)+g(X, \mathrm{t} V)=0 .
$$

We may state

THEOREM 8. Let $N$ be a submanifold of the a.ct.m. manifold $N_{0}$, tangent to the contact vector $(\xi \in T(N))$. Then $N$ is a contact $C R$ submanifold if and only if $\mathrm{FP}=0$. If this is the case $\mathscr{D}_{1}:=\operatorname{Ker}\left(\mathrm{I}+\mathrm{P}^{2}-\eta \otimes \xi\right)$ and $\mathscr{D}_{2}:=\operatorname{Ker}\left(\mathrm{I}+\mathrm{P}^{2}\right)$ are $\phi$-invariant and $\xi \in \mathscr{D}_{1}, \xi \in \mathscr{D}_{2}^{\perp}$.

This was first proved by K. Yano \& M. Kon (cf. [29]) though for a Sasakian ambient space only. The proof is verbatim repetition of the arguments in [29], p. 51 .

Let us prove Theorem 1. To this end, let $H(N)$ be the orthogonal complement of $\mathbf{R} \xi \xi$ in $\mathscr{D}$, and $H(N)^{\perp}$ the orthogonal complement of $H(N)$ in $T(N)$. 
Set $m:=\operatorname{dim}_{\mathbf{R}} H(N)_{x}$ and $k:=\operatorname{dim}_{\mathbf{R}} H(N)_{x}^{\perp}, x \in N . \quad$ Let $X \in H(N)$ and $Z \in$ $H(N)^{\perp}$. As

$$
H(N)^{\perp}=\mathscr{D}^{\perp} \oplus \mathbf{R} \imath \xi
$$

it follows that $Z=W+\lambda l \xi$, for some $W \in \mathscr{D}^{\perp}$ and $\lambda \in C^{\infty}(N)$. Then (by $\left.g\left(\mathscr{D}, \mathscr{D}^{\perp}\right)=0\right)$

$$
g(\phi X, Z)=\lambda g(\phi X, l \xi)=\lambda \eta(\phi X)=0 .
$$

Here $\eta=i^{*} \eta_{0}$. Thus $\phi X \in H(N)$, that is $\phi H(N) \subseteq H(N)$. This proves that $H(N)$ is $\phi$-invariant. To show that $H(N)^{\perp}$ is $\phi$-anti-invariant, let $Z \in H(N)^{\perp}$ and $X \in T(N)$. Then, using the decomposition (2.7), $\phi W \in T(N)^{\perp}$, and

$$
0=\phi \xi=\phi l \xi+\phi l^{\perp} \xi \in \mathscr{D} \oplus T(N)^{\perp} \Rightarrow \phi l \xi=0,
$$

we have

$$
g_{0}(\phi Z, X)=g_{0}(\phi(W+\lambda l \xi), X)=g_{0}(\phi W, X)=0 .
$$

Therefore $\phi Z \in T(N)^{\perp}$, that is $\phi H(N)^{\perp} \subseteq T(N)^{\perp}$. Let $X \in H(N)$. Then

$$
\phi^{2} X=-X+\eta(X) \xi=-X
$$

hence $\xi \in H(N)^{\perp}$. In particular, the real dimension of $H(N)_{x}, x \in N$, must be even, i.e. $m=2 n$. At this point we may check that $T_{1,0}(N):=[T(N) \otimes \mathbf{C}] \cap$ $T_{1,0}\left(N_{0}\right)$ is a CR structure on $N$. Here $T_{1,0}\left(N_{0}\right):=\left\{A-i \phi A: A \in \operatorname{Ker}\left(\eta_{0}\right)\right\}$ is the CR structure (of type $(l, 1)$, with $\operatorname{dim} N_{0}=2 l+1$ ) of $N_{0}$. To this end, note that

$$
T_{1,0}(N)=\operatorname{Eigen}\left(J^{\mathbf{C}}, i\right) .
$$

Here $J^{\mathbf{C}}$ is the $\mathbf{C}$-linear extension of $J:=\left.\phi\right|_{H(N)}$ to $H(N) \otimes \mathbf{C}$. Indeed, let $X-i J X \in \operatorname{Eigen}\left(J^{\mathbf{C}}, i\right), X \in H(N)$. As $H(N) \subseteq \operatorname{Ker}\left(\eta_{0}\right)$, it follows that $X-$ $i J X \in T_{1,0}(N)$ and then

$$
\operatorname{Eigen}\left(J^{\mathbf{C}}, i\right) \subseteq[T(N) \otimes \mathbf{C}] \cap T_{1,0}\left(N_{0}\right) .
$$

To check the opposite inclusion, let $A-i \phi A \in T_{1,0}\left(N_{0}\right) \cap[T(N) \otimes \mathbf{C}]$. Then $A-i \phi A=X+i Y$, for some $X, Y \in T(N)$. It follows that $A=X$ and $\phi A=-Y$, that is $A \in T(N) \cap \operatorname{Ker}\left(\eta_{0}\right)$. Therefore

$$
A \in(\mathbf{R} l \xi)^{\perp}=\operatorname{Ker}(\eta) \subset T(N) .
$$

Here $(\mathbf{R} l \xi)^{\perp}$ is the orthogonal complement of $\mathbf{R}_{l} \xi$ in $T(N)$. Let $E(N)$ be the orthogonal complement of $\mathbf{R} l \xi$ in $H(N)^{\perp}$, so that

$$
H(N)^{\perp}=E(N) \oplus \mathbf{R} l \xi, \quad \mathscr{D}^{\perp}=E(N) \oplus \mathbf{R} l^{\perp} \xi .
$$

Then

$$
(\mathbf{R} l \xi)^{\perp}=H(N) \oplus E(N) .
$$

Now (2.9) and (2.10) yield $A=A_{H}+A_{E}$, for some $A_{H} \in H(N)$ and $A_{E} \in E(N)$. Therefore 


$$
-Y=\phi A=\phi A_{H}+\phi A_{E}
$$

hence $\phi A_{H}=-Y$ and $\phi A_{E}=0$ (because of $\phi A_{E} \in \phi \mathscr{D}^{\perp} \subseteq T(N)^{\perp}$ ). It follows that

$$
0=\phi^{2} A_{E}=-A_{E}+\eta_{0}\left(A_{E}\right) \xi=-A_{E} .
$$

Summing up, we have $A_{E}=0$, that is $A=A_{H} \in H(N)$. Then $A-i \phi A \in$ $\operatorname{Eigen}\left(J^{\mathbf{C}}, i\right)$ and $(2.8)$ is proved. In particular (by $\left.(2.8)\right) T_{1,0}(N)$ has constant rank $n$. Moreover, by the very definition of $T_{1,0}(N)$

$$
T_{1,0}(N) \cap \overline{T_{1,0}(N)} \subseteq T_{1,0}\left(N_{0}\right) \cap T_{0,1}\left(N_{0}\right)=(0) .
$$

Both $T(N) \otimes \mathbf{C}$ and $T_{1,0}\left(N_{0}\right)$ are formally integrable and then so is $T_{1,0}(N)$. Q.e.d.

\subsection{Real orientable hypersurfaces in a.ct.m. manifolds}

As an application of Theorem 1 we look at real orientable hypersurfaces in an a.ct.m. manifold whose underlying almost contact structure is normal. We may state

THEOREM 9. Let $N_{0}$ be a $(2 n+1)$-dimensional a.ct.m. manifold, whith $\left(\phi, \xi, \eta_{0}\right)$ normal. Let $N$ be a real orientable hypersurface in $N_{0}$, tangent to the contact vector $(\xi \in T(N))$. Let $v$ be a unit normal field on $N$. Then $U:=\phi v$ is tangent to $N$. Let $H(N)$ be the orthogonal complement of $\mathbf{R} U \oplus \mathbf{R} \xi$ in $T(N)$. Then $(N, H(N))$ is a contact $C R$ submanifold of $N_{0}$. Consequently $T_{1,0}(N):=$ $\{X-i \phi X: X \in H(N)\}$ is a CR structure of type $(n-1,2)$.

Proof. Note that $U$ and $\xi$ are orthogonal. Next

$$
\begin{aligned}
g_{0}(U, v) & =g_{0}(\phi v, v)=g_{0}\left(\phi^{2} v, \phi v\right)+\eta_{0}(\phi v) \eta_{0}(v) \\
& =g_{0}\left(-v+\eta_{0}(v) \xi, U\right)=-g_{0}(v, U),
\end{aligned}
$$

hence $U$ is tangent to $N$. Let $X \in H(N)$. We have

$$
g_{0}(\phi X, v)=-g_{0}(X, \phi v)=-g_{0}(X, U)=0 .
$$

It follows that $\phi X \in T(N)$, i.e. $\phi H(N) \subseteq T(N)$. Moreover

$$
\begin{gathered}
g(\phi X, U)=g_{0}(\phi X, \phi v)=g_{0}(X, v)-\eta_{0}(X) \eta_{0}(v)=0, \\
g(\phi X, \xi)=\eta(\phi X)=0 .
\end{gathered}
$$

Thus $\phi X \in H(N)$, that is $H(N)$ is $\phi$-invariant. Now, let $Y \in H(N)^{\perp}=$ $\mathbf{R} U \oplus \mathbf{R} \xi$. As $\phi \xi=0$ and $\phi U=-v \in T(N)^{\perp}$ it follows that $\phi Y \in T(N)^{\perp}$, that is $H(N)^{\perp}$ is $\phi$-anti-invariant. $\quad$ Q.e.d.

By Theorem 9, given a smooth domain $\Omega_{0} \subset S^{2 n+1}$, its boundary $N=\partial \Omega_{0}$ is a CR manifold of CR codimension 2, in contrast with domains in $\mathbf{C}^{n}$ (where the $\mathrm{CR}$ codimension and the codimension of the boundary are both 1). A geometric study of CR manifolds of CR codimension 2 was begun by R. Mizner, 
[17] (using Cartan connections); the case of real hypersurfaces in Sasakian manifolds is not considered. As to analysis on domains in a CR manifold, the subject starts with the Hartogs-Bochner theorem on a CR manifold, due to G. M. Henkin, [12]. He proves that, given a 1-concave generic CR submanifold $M \subset \mathbf{C}^{n}$, a bounded domain $\Omega \subset M$ and a smooth domain $\Omega_{0}$ contained in $\Omega$ and such that $\Omega \backslash \Omega_{0}$ is connected, each CR function $f \in C R^{\infty}\left(\partial \Omega_{0}\right)$ extends to a function $F \in C^{\infty}\left(\bar{\Omega}_{0}\right) \cap C R^{\infty}\left(\Omega_{0}\right)$. The assumption that the diameter of $\Omega_{0}$ is "sufficiently small" (adopted in [12]) was removed subsequently by C. LaurentThiébaut, [14]. The assumption that $M$ is 1-concave concerns the Levi form and the geometry of the boundary $N=\partial \Omega_{0}$ is not studied. However, for a smooth domain $\Omega_{0} \subset S^{2 n+1}$, it is natural to expect that the geometry of the CR manifold $N=\partial \Omega_{0}$ is tied to the function theory on $\Omega_{0}$, and conversely. In the next section we look at the equations induced on $N$ by the tangential CauchyRiemann equations of $S^{2 n+1}$. Note that the Hartogs-Bochner theorem (cf. op. cit.) holds on $M=S^{2 n+1} \subset \mathbf{C}^{n+1}$ (as the Levi form of $M$ is positive definite). Finally, to underline the novelty of the situation, note that the pseudohermitian tools (e.g. the Tanaka-Webster connection, the Chern-Moser tensor, etc., cf. [21] and [24]) are not available in the CR codimension 2 case.

\section{Traces of $\mathbf{C R}$ functions on contact $\mathrm{CR}$ submanifolds}

\subsection{Traces of $\mathbf{C R}$ functions}

Let $\left(N_{0},\left(\phi, \xi, \eta_{0}, g_{0}\right)\right)$ be a $(2 n+1)$-dimensional a.ct.m. manifold with $\left(\phi, \xi, \eta_{0}\right)$ normal. Then $T_{1,0}\left(N_{0}\right)=\left\{X-i \phi X: X \in \operatorname{Ker}\left(\eta_{0}\right)\right\}$ is a CR structure of type $(n, 1)$ on $N_{0}$. For a CR manifold $M$, let $C R^{k}(M)$ be the space of all $C R$ funtions of class $C^{k}, k \in \mathbf{N} \cup\{\infty, \omega\}$, i.e. solutions $f$ of class $C^{k}$ to the tangential Cauchy-Riemann equations (1.4). Then

$$
C R^{k}\left(N_{0}\right)=\left\{f \in C^{k}\left(N_{0}\right):(X+i \phi X) f=0, \forall X \in \operatorname{Ker}\left(\eta_{0}\right)\right\} .
$$

Let $(N, \mathscr{D})$ be a contact $\mathrm{CR}$ submanifold of $N_{0}$. By the very definition $T_{1,0}(N) \subset T_{1,0}\left(N_{0}\right)$, hence the restriction $C^{\infty}\left(N_{0}\right) \rightarrow C^{\infty}(N)$ descends to a map $C R^{\infty}\left(N_{0}\right) \rightarrow C R^{\infty}(N)$. When $N$ is a real hypersurface, for any $x \in N$ there is an open neighborhood $U \subseteq N_{0}$ and a real valued function $\rho \in C^{\infty}(U)$ such that $N \cap U=\{x \in U: \rho(x)=0\}$. Let $\left\{T_{\alpha}\right\}$ be a (local) frame of $T_{1,0}\left(N_{0}\right)$ on $U$ and set $V:=\left\{x \in U: T_{n}(\rho)_{x} \neq 0\right\}$. Then the trace $u$ on $V$ of $f \in C R^{\infty}\left(N_{0}\right)$ is a solution to $T_{\bar{j}}(u)-\left(T_{\bar{j}}(\rho) / T_{\bar{n}}(\rho)\right) T_{\bar{n}}(u)=0,1 \leq j \leq n-1$. For instance, let $N_{0}=\mathbf{H}_{n}=\mathbf{C}^{n} \times \mathbf{R}$ be the Heisenberg group with the CR structure spanned by $T_{\alpha}=\partial / \partial z^{\alpha}+i z^{\alpha}(\partial / \partial t), 1 \leq \alpha \leq n$ (the Lewy operators). Consider the submanifold $N=\Sigma^{2 n}:=\left\{x \in \mathbf{H}_{n}:|x|=1\right\}$, where $|x|:=\left(|z|^{4}+t^{2}\right)^{1 / 4}$ is the Heisenberg norm. $\quad \mathbf{H}_{n}$ is a Sasakian manifold in a natural way hence Theorem 8 applies. As

$$
T_{\alpha}(\rho)=\frac{1}{2}|x|^{-3} \bar{z}^{\alpha} \bar{\phi}, \quad \phi(z, t):=|z|^{2}-i t \in C R^{\infty}\left(\mathbf{H}_{n}\right),
$$

each $u \in C R^{\infty}\left(\Sigma^{2 n}\right)$ is a solution to $T_{\bar{j}}(u)-\left(z^{j} / z^{n}\right) T_{\bar{n}}(u)=0$. Therefore, the tangential Cauchy-Riemann equations on $\Sigma^{2 n} \cap\left\{z^{n} \neq 0\right\}$ read 


$$
\frac{\partial u}{\partial \bar{z}^{j}}-\frac{z^{j}}{z^{n}} \frac{\partial u}{\partial \bar{z}^{n}}=0
$$

For a contact CR submanifold $N$ of a Sasakian manifold $N_{0}$, it is an open problem whether $C R^{1}\left(N_{0}\right) \rightarrow C R^{1}(N)$ is an epimorphism (the local version of the same problem is open, as well). A partial answer is provided by Theorem 2 .

\subsection{The Boothby-Wang fibration}

Let $N_{0}$ be a Sasakian manifold. A local chart $(U, \varphi)$ of $N_{0}$ is cubic (of side $2 a$ and center $\left.x_{0} \in N_{0}\right)$ if $\varphi\left(x_{0}\right)=0$ and $\varphi(U)=\left\{\left(t^{1}, \ldots, t^{2 n+1}\right):\left|t^{j}\right|<a\right.$, $1 \leq j \leq 2 n+1\}$. Let $\left(U, \varphi=\left(x^{1}, \ldots, x^{2 n+1}\right)\right)$ be a cubic chart of $N_{0}$. Let $1 \leq$ $p \leq 2 n+1$ and $t=\left(t^{p+1}, \ldots, t^{2 n+1}\right)$ such that $\left|t^{p+j}\right|<a, \quad 1 \leq j \leq 2 n-p+1$. Then $\Sigma_{t}:=\left\{y \in U: x^{p+j}(y)=t^{p+j}, 1 \leq j \leq 2 n-p+1\right\}$ is a $p$-dimensional slice of $(U, \varphi)$. The contact vector $\xi$ is regular if $N_{0}$ admits a $C^{\infty}$ atlas $\{(U, \varphi)\}$ such that the intersection of $U$ with any maximal integral curve of $\xi$ is a 1-dimensional slice of $(U, \varphi)$. By a result of $\mathrm{R}$. Palais (cf. [20]) if $\xi$ is regular the quotient space $N_{0} / \xi$ admits a natural structure of a $C^{\infty}$ manifold such that the projection $\pi_{0}: N_{0} \rightarrow M_{0}:=N_{0} / \xi$ is $C^{\infty}$. The contact vector of the standard sphere $S^{2 n+1}$ is regular. By a result of W. M. Boothby \& H. C. Wang (cf. [7]), if $N_{0}$ is a compact Sasakian manifold with $\xi$ regular, there is a free action of $S^{1}$ on $N_{0}$ making $N_{0}$ into a $S^{1}$-principal bundle over $M_{0}=N_{0} / \xi$, with projection $\pi_{0}$ : $N_{0} \rightarrow M_{0}$. The contact vector $\xi$ is tangent to the $S^{1}$-orbits and $\eta_{0}$ is a connection 1-form. Moreover, the Sasakian structure of $N_{0}$ gives rise to a complex structure $J$ and a Kählerian metric $G_{0}$ on $M_{0}$ given by

$$
(J X)^{\uparrow}=\phi X^{\uparrow}, \quad G_{0}(X, Y)^{v}=g_{0}\left(X^{\uparrow}, Y^{\uparrow}\right), \quad X, Y \in T\left(M_{0}\right) .
$$

Here $X^{\uparrow}$ is the horizontal lift of $X$ with respect to $\eta_{0}$. Also, if $f \in C^{\infty}\left(M_{0}\right)$ we set $f^{v}:=f \circ \pi_{0}$ (the vertical lift of $f$ ). Then $\pi_{0}:\left(N_{0}, g_{0}\right) \rightarrow\left(M_{0}, G_{0}\right)$ is a Riemannian submersion and one may apply the results of B. O'Neill, [19]. In particular, if $h: T\left(N_{0}\right) \rightarrow H\left(N_{0}\right)$ is the projection associated to the direct sum decomposition $T\left(N_{0}\right)=H\left(N_{0}\right) \oplus \operatorname{Ker}\left(d \pi_{0}\right)$ then $h \nabla_{X^{\uparrow}}^{0} Y^{\uparrow}$ is $\pi_{0}$-related to $\nabla_{X}^{M_{0}} Y$. Here $\nabla^{0}$ and $\nabla^{M_{0}}$ are the Levi-Civita connections of $\left(N_{0}, g_{0}\right)$ and $\left(M_{0}, G_{0}\right)$. Consequently (cf. K. Yano \& M. Kon, [29], p. 100)

$$
\nabla_{X^{\dagger}}^{0} Y^{\uparrow}=\left(\nabla_{X}^{M_{0}} Y\right)^{\uparrow}-G_{0}(X, J Y)^{v} \xi, \quad X, Y \in \mathscr{X}\left(M_{0}\right) .
$$

For the sake of completeness we give a short proof of (3.1). We have $\nabla_{X^{\uparrow}}^{0} Y^{\uparrow}=A+\lambda \xi$, for some $A \in H\left(N_{0}\right)$ and $\lambda \in C^{\infty}\left(N_{0}\right)$. Then

$$
\left(d \pi_{0}\right) A=\left(d \pi_{0}\right) \nabla_{X^{\uparrow}}^{0} Y^{\uparrow}=\nabla_{X}^{M_{0}} Y=\left(d \pi_{0}\right)\left(\nabla_{X}^{M_{0}} Y\right)^{\uparrow}
$$

i.e. $A-\left(\nabla_{X}^{M_{0}} Y\right)^{\uparrow} \in \operatorname{Ker}\left(d \pi_{0}\right) \cap H\left(N_{0}\right)=(0)$. Recall that, on a Sasakian manifold $\nabla_{Z}^{0} \xi=-\phi Z$. On the other hand $g_{0}\left(Y^{\uparrow}, \xi\right)=\eta_{0}\left(Y^{\uparrow}\right)=0$, hence

$$
\begin{aligned}
\lambda & =g_{0}\left(\nabla_{X^{\uparrow}}^{0} Y^{\uparrow}, \xi\right)=X^{\uparrow}\left(g_{0}\left(Y^{\uparrow}, \xi\right)\right)-g_{0}\left(Y^{\uparrow}, \nabla_{X^{\dagger}}^{0} \xi\right) \\
& =g_{0}\left(Y^{\uparrow}, \phi X^{\uparrow}\right)=g_{0}\left(Y^{\uparrow},(J X)^{\uparrow}\right)=G_{0}(Y, J X)^{v}=-G_{0}(X, J Y)^{v} . \quad \text { Q.e.d. }
\end{aligned}
$$




\subsection{Cauchy-Riemann fibrations}

Let $N_{0}$ be a compact Sasakian manifold with $\xi$ regular and $S^{1} \rightarrow N_{0} \rightarrow$ $M_{0}=N_{0} / \xi$ the corresponding Boothby-Wang fibration. Let $j: M \subset M_{0}$ be a submanifold and $N:=\pi_{0}^{-1}(M)$, so that $S^{1} \rightarrow N \stackrel{\pi}{\rightarrow} M$ is a principal subbundle of $S^{1} \rightarrow N_{0} \rightarrow M_{0}$ (with $\pi:=\left.\pi_{0}\right|_{N}$ ). We adopt the following notations: $B$ (respectively $h$ ) and $A_{V}$ (respectively $a_{W}$ ) are the second fundamental form of $j$ : $M \subset M_{0}$ (respectively of $i: N \subset N_{0}$ ) and the Wiengarten operator corresponding to the normal section $V \in \Gamma^{\infty}(\mathscr{V}(j)), \mathscr{V}(j)=T(M)^{\perp}$, (respectively to $W \in$ $\left.\Gamma^{\infty}(\mathscr{V}(i)), \mathscr{V}(i)=T(N)^{\perp}\right)$. Moreover $g=i^{*} g_{0}$ and $G=j^{*} G_{0}$ are the induced metrics on $N$ and $M$ and $\nabla^{\perp}, \nabla^{\perp, M}$ are the normal connections in $\mathscr{V}(i)$ and $\mathscr{V}(j)$. We also set $\eta=i^{*} \eta_{0}$ and $\mathscr{H}:=\operatorname{Ker}(\eta)=\operatorname{Ker}\left(\eta_{0}\right) \cap T(N)=H\left(N_{0}\right) \cap$ $T(N) . \quad \mathscr{H}$ is a connection-distribution in the principal bundle $S^{1} \rightarrow N \rightarrow M$. Clearly $\xi$ is tangent to $N$ and spans $\operatorname{Ker}(d \pi)$. Moreover

$$
T(M)^{\uparrow}=\mathscr{H}, \quad\left(T(M)^{\perp}\right)^{\uparrow}=T(N)^{\perp} .
$$

The Gauss formulae of $M \subset M_{0}$ and $N \subset N_{0}$

$$
\nabla_{X}^{M_{0}} Y=\nabla_{X}^{M} Y+B(X, Y), \quad \nabla_{X^{\uparrow}}^{0} Y^{\uparrow}=\nabla_{X^{\uparrow}} Y^{\uparrow}+h\left(X^{\uparrow}, Y^{\uparrow}\right),
$$

together with (3.1), lead to

$$
\begin{aligned}
\nabla_{X^{\uparrow}} Y^{\uparrow}+h\left(X^{\uparrow}, Y^{\uparrow}\right) & =\left(\nabla_{X}^{M_{0}} Y\right)^{\uparrow}-G_{0}(X, J Y)^{v} \xi \\
& =\left(\nabla_{X}^{M} Y\right)^{\uparrow}+B(X, Y)^{\uparrow}-G\left(X, \mathrm{P}_{M} Y\right)^{v} \xi
\end{aligned}
$$

where $\mathrm{P}_{M} X:=\tan (J X), X \in T(M)$. We obtain

$$
\begin{gathered}
\nabla_{X^{\uparrow}} Y^{\uparrow}=\left(\nabla_{X}^{M} Y\right)^{\uparrow}-G\left(X, \mathrm{P}_{M} Y\right)^{v} \xi, \\
h\left(X^{\uparrow}, Y^{\uparrow}\right)=B(X, Y)^{\uparrow} .
\end{gathered}
$$

The Weingarten formulae of $M \subset M_{0}$ and $N \subset N_{0}$

$$
\nabla_{X}^{M_{0}} V=-A_{V} X+\nabla_{X}^{\perp, M} V, \quad \nabla_{X^{\uparrow}}^{0} V^{\uparrow}=-a_{V^{\uparrow}} X^{\uparrow}+\nabla_{X^{\uparrow}}^{\perp} V^{\uparrow},
$$

together with (3.1) lead to

$$
\begin{aligned}
-a_{V^{\uparrow}} X^{\uparrow}+\nabla_{X^{\uparrow}}^{\perp} V^{\uparrow} & =\left(\nabla_{X}^{M_{0}} V\right)^{\uparrow}-G_{0}(X, J V)^{v} \xi \\
& =-\left(A_{V} X\right)^{\uparrow}+\left(\nabla_{X}^{\perp, M} V\right)^{\uparrow}-G\left(X, \mathrm{t}_{M} V\right)^{v} \xi
\end{aligned}
$$

where $\mathrm{t}_{M} V:=\tan (J V), V \in T(M)^{\perp}$. We obtain

$$
\begin{gathered}
a_{V^{\uparrow}} X^{\uparrow}=\left(A_{V} X\right)^{\uparrow}+G\left(X, \mathrm{t}_{M} V\right)^{v} \xi, \\
\nabla_{X^{\dagger}}^{\perp} V^{\uparrow}=\left(\nabla_{X}^{\perp, M} V\right)^{\uparrow} .
\end{gathered}
$$

Set $\mathrm{F}_{M} X:=\operatorname{nor}(J X)$ and $\mathrm{f}_{M} V:=\operatorname{nor}(J V)$. Then

$$
\begin{aligned}
\mathrm{P} X^{\uparrow}=\left(\mathrm{P}_{M} X\right)^{\uparrow}, & \mathrm{F} X^{\uparrow}=\left(\mathrm{F}_{M} X\right)^{\uparrow}, \\
\mathrm{t} V^{\uparrow}=\left(\mathrm{t}_{M} V\right)^{\uparrow}, & \mathrm{f} V^{\uparrow}=\left(\mathrm{f}_{M} V\right)^{\uparrow} .
\end{aligned}
$$


By a result of K. Yano \& M. Kon (cf. Theor. 2.1 in [28], p. 375) $N$ is a contact CR submanifold of $N_{0}$ if and only if $M$ is a CR submanifold of $M_{0}$. If this is the case, that is $N$ is both a contact CR submanifold and the total space of a $S^{1}$-principal bundle over a CR submanifold $M$, we call $S^{1} \rightarrow N \rightarrow M$ a CauchyRiemann $(C-R)$ circle bundle. Given a C-R circle bundle $S^{1} \rightarrow N \rightarrow M$, the distribution $\mathscr{D}:=\operatorname{Ker}\left(\mathrm{I}+\mathrm{P}^{2}-\eta \otimes \xi\right)$ is $\phi$-invariant and $\mathscr{D}^{\perp}$ (the orthogonal complement of $\mathscr{D}$ in $T(N))$ is $\phi$-anti-invariant. Also $H(M):=\operatorname{Ker}\left(\mathrm{I}+\mathrm{P}_{M}^{2}\right)$ is $J$-invariant, while $H(M)^{\perp}$ (the orthogonal complement of $H(M)$ in $T(M)$ ) is $J$-anti-invariant.

Let us consider a foliated manifold $(M, \mathscr{F})$, with the foliation $\mathscr{F}$. A subset $A \subseteq M$ is saturated if $A$ is a union of leaves of $\mathscr{F}$, i.e. every leaf of $\mathscr{F}$ intersecting $A$ is contained in $A$. A smooth function $f: M \rightarrow \mathbf{C}$ is a basic function if $X(f)=0$ for every $X \in T(\mathscr{F})$.

The total space $N_{0}$ of the Boothby-Wang fibration $S^{1} \rightarrow N_{0} \rightarrow M_{0}$ is a foliated manifold, in a natural way. Indeed, the vertical distribution $\operatorname{Ker}\left(d \pi_{0}\right)=$ $\mathbf{R} \xi$ is integrable and so, by the Frobenius theorem, there is a foliation $\mathscr{V}_{0}$ on $N_{0}$ tangent to $\operatorname{Ker}\left(d \pi_{0}\right)$. The leaves of $\mathscr{V}_{0}$ are the maximal integral curves of the contact vector field $\xi$. If $S^{1} \rightarrow N \rightarrow M$ is a C-R (circle) bundle, then $N$ is a saturated subset of $\left(N_{0}, \mathscr{V}_{0}\right)$. Therefore $\mathscr{V}_{0}$ induces a foliation $\mathscr{V}$ on $N$. We shall need the following lemma

Lemma 1. Let $S^{1} \rightarrow N \rightarrow M$ be a $C$ - $R$ fibration. Consider the invariant distributions $\mathscr{D}=\operatorname{Ker}\left(\mathrm{I}+\mathrm{P}^{2}-\eta \otimes \xi\right)$ and $H(M)=\operatorname{Ker}\left(\mathrm{I}+\mathrm{P}_{M}^{2}\right)$. Then $(N, \mathscr{D})$ is generic if and only if $(M, H(M))$ is generic.

Proof. Note that $\xi \in \mathscr{D}$. Let $H(N)$ be the orthogonal complement of $\mathbf{R} \xi$ in $\mathscr{D}$, so that $\mathscr{D}=H(N) \oplus \mathbf{R} \xi$. Moreover $T(N)=\mathscr{D} \oplus \mathscr{D}^{\perp}$ and $T(N)=$ $\mathscr{H} \oplus \mathbf{R} \xi$ imply

$$
\mathscr{H}=H(N) \oplus \mathscr{D}^{\perp} .
$$

Hence it is enough to prove that

$$
H(M)^{\uparrow}=H(N) .
$$

Indeed (3.10)-(3.9) yield $\left(H(M)^{\perp}\right)^{\uparrow}=\mathscr{D}^{\perp}$ and lemma 1 is proved $\left(\right.$ as $\left(T(M)^{\perp}\right)^{\uparrow}$ $\left.=T(N)^{\perp}\right)$. Now we prove (3.10). Let $X \in H(M)$. Then

$$
\left(\mathrm{I}+\mathrm{P}^{2}-\eta \otimes \xi\right) X^{\uparrow}=\left[\left(\mathrm{I}+\mathrm{P}_{M}^{2}\right) X\right]^{\uparrow}-\eta\left(X^{\uparrow}\right) \xi=0
$$

as $X \in \operatorname{Ker}\left(\mathrm{I}+\mathrm{P}_{M}^{2}\right)$ and $X^{\uparrow} \in H(M)^{\uparrow} \subset T(M)^{\uparrow}=\mathscr{H}=\operatorname{Ker}(\eta)$. It follows that $X^{\uparrow} \in \operatorname{Ker}\left(\mathrm{I}+\mathrm{P}^{2}-\eta \otimes \xi\right)=H(N)$ and so $H(M)^{\uparrow} \subseteq H(N)$. The opposite inclusion is a bit more difficult. Let $\tilde{X} \in H(N) \subset \mathscr{H}=T(M)^{\uparrow}$. Then $\tilde{X}=X^{\uparrow}$ for some $X \in T(M)$ and

$$
0=\left(\mathrm{I}+\mathrm{P}^{2}-\eta \otimes \xi\right) \tilde{X}=\left[\left(\mathrm{I}+\mathrm{P}_{M}^{2}\right) X\right]^{\uparrow}
$$

yields $X \in H(M)$, i.e. $\tilde{X} \in H(M)^{\uparrow}$. Q.e.d. 
Now we may prove Theorem 2. Let $S^{1} \rightarrow N \rightarrow M$ be a C-R fibration over a compact, connected, real analytic CR submanifold. Let us assume that $N$ is generic and proper. First, let us show that if $(N, \mathscr{D})$ is a contact $C R$ submanifold of an a.ct.m. manifold $\left(N_{0},\left(\phi, \xi, \eta_{0}, g_{0}\right)\right)$ such that $\xi \in \mathscr{D}$, then $\mathscr{D}=$ $\operatorname{Ker}\left(\mathrm{I}+\mathrm{P}^{2}-\eta \otimes \xi\right)$. Indeed, let $X \in \mathscr{D}$ be orthogonal to $\xi$. Then

$$
\left(\mathrm{I}+\mathrm{P}^{2}-\eta \otimes \xi\right) X=\left(\mathrm{I}+\mathrm{P}^{2}\right) X=0,
$$

as $\mathrm{P}$ is a complex structure in $H(N)$. Conversely, let us consider $X \in$ $\operatorname{Ker}\left(\mathrm{I}+\mathrm{P}^{2}-\eta \otimes \xi\right)$, orthogonal to $\xi$. As $\mathrm{P}(T(N)) \subseteq \mathscr{D}$ and $\mathrm{t}\left(T(N)^{\perp}\right) \subseteq \mathscr{D}^{\perp}$, one has $l=-\mathrm{P}^{2}+\eta \otimes \xi$. Then

$$
0=\left(\mathrm{I}+\mathrm{P}^{2}-\eta \otimes \xi\right) X=X-{ }_{\imath} X
$$

hence $X \in \mathscr{D}$. Q.e.d.

Let $(N, \mathscr{D})$ be a contact $\mathrm{CR}$ submanifold of an almost contact manifold $N_{0}$, tangent to $\xi$ and generic. Then $\xi$ is transverse to $\mathscr{D}^{\perp}$. Indeed, if there is $x_{0} \in N$ such that $(l \xi)_{x_{0}}=0$, then $H(N)_{x_{0}}^{\perp}=\mathscr{D}_{x_{0}}^{\perp}$ hence (by genericity) $k=\operatorname{codim} N=$ $\operatorname{dim} N_{0}-(2 n+k)$, a contradiction. It follows that, whenever $N$ is generic, one has $(l \xi)_{x} \neq 0$ for any $x \in N$ and then the dimension of the ambient space must be $\operatorname{dim} N_{0}=2(n+k)-1$ (as in the hypothesis of Theorem 2).

Lemma 1 implies that $M$ is a generic proper CR submanifold of $\mathbf{C P}^{n+k-1}$. By a result of R. O. Wells, [26], there is a connected set $Q_{M} \subset \mathbf{C P}^{n+k-1}$ such that $Q_{M} \supset M$ and there is a submanifold $M^{\prime} \subset \mathbf{C P}^{n+k-1}$ of dimension $2 n+k$ such that $Q_{M} \supset M^{\prime}$ and each holomorphic function on a neighborhood of $M$ extends to a holomorphic function on a neighborhood of $Q_{M}$. Set $Q:=$ $\pi_{0}^{-1}\left(Q_{M}\right)$. Clearly $Q$ is a saturated set (i.e. a union of leaves of the vertical foliation on $S^{2(n+k)-1}$ ) and $Q \supset N, Q \supset N^{\prime}$, where $N^{\prime}:=\pi_{0}^{-1}\left(M^{\prime}\right)$. As $N$ is locally diffeomorphic to $M \times S^{1}$ and $M$ is real analytic, it follows that $N$ is real analytic as well. Let $u \in C R^{\omega}(N)$ be a basic (i.e. constant along the leaves of $\mathscr{V}) \mathrm{CR}$ function. There is a $C^{\omega}$ function $\tilde{u}: M \rightarrow \mathbf{C}$ such that $\tilde{u} \circ \pi=u$, where $\pi:=\left.\pi_{0}\right|_{N}$. For any $v \in C^{1}(M)$ we have

$$
\pi^{*} \bar{\partial}_{M} v=\bar{\partial}_{N}(v \circ \pi) .
$$

In particular $v \in C R^{1}(M)$ if and only if $v \circ \pi \in C R^{1}(N)$. Here $\bar{\partial}_{M}$ and $\bar{\partial}_{N}$ are, respectively, the tangential Cauchy-Riemann operators on $M$ and $N$. We postpone the proof of (3.11). To end the proof of Theorem 2, note that (by (3.11)) $0=\bar{\partial}_{N} u=\pi^{*} \bar{\partial}_{M} \tilde{u}$ hence $\tilde{u} \in C R^{\omega}(M)$. By the Tomassini theorem, there is an open neighborhood $U_{0}$ of $M$ in $\mathbf{C P}^{n+k-1}$ and there is a unique holomorphic function $f_{0} \in \mathcal{O}\left(U_{0}\right)$ such that $\left.f_{0}\right|_{M}=\tilde{u}$. By the aforementioned result of R. O. Wells (cf. [26]) there is a neighborhood $\tilde{U}$ of $Q_{M}$ in $\mathbf{C P}^{n+k-1}$ and there is a holomorphic function $\tilde{f} \in \mathcal{O}(\tilde{U})$ which extends $f_{0}$, i.e. $\left.\tilde{f}\right|_{\tilde{U} \cap U_{0}}=f_{0}$. Set $U:=$ $\pi_{0}^{-1}(\tilde{U})$ and $f:=\tilde{f} \circ \pi_{0} \in C^{\omega}(U)$. Then $U$ is open and saturated in $S^{2(n+k)-1}$ and $U \supset Q$. At this point, we need the identity

$$
\pi_{0}^{*} \bar{\partial} v=\bar{\partial}_{S^{2(n+k)-1}}\left(v \circ \pi_{0}\right),
$$


for any $v \in C^{1}\left(\mathbf{C P}^{n+k-1}\right)$. Here $\bar{\partial}$ is the Cauchy-Riemann operator on $\mathbf{C P}^{n+k-1}$. By (3.12) one has $\bar{\partial}_{S^{2(n+k)-1}} f=\pi_{0}^{*} \bar{\partial} \tilde{f}=0$, i.e. $f \in C R^{\omega}(U)$. Finally $f \circ i=\tilde{f} \circ$ $\pi_{0} \circ i=\tilde{f} \circ j \circ \pi=f_{0} \circ j \circ \pi=\tilde{u} \circ \pi=u$, i.e. $\left.f\right|_{N}=u$. It remains that we prove (3.11) and (3.12). If $(M, \mathscr{D})$ is a CR submanifold of a Hermitian manifold, the tangential Cauchy-Riemann operator is the differential operator $\bar{\partial}_{M}: C^{\infty}(M) \rightarrow$ $\Omega^{0,1}(M)$ defined as follows. A complex valued 1-form $\omega \in \Omega^{1}(M)=\Gamma^{\infty}\left(T^{*}(M)\right.$ $\otimes \mathbf{C})$ is a $(0,1)$-form if $\left.T_{1,0}(M)\right\rfloor \omega=0$ and $\left.\mathscr{D}^{\perp}\right\rfloor \omega=0$. Let $\bigwedge^{0,1}(M)$ be the vector bundle of all $(0,1)$-forms on $M$ and $\Omega^{0,1}(M):=\Gamma^{\infty}\left(\bigwedge^{0,1}(M)\right)$. If $u$ : $M \rightarrow \mathbf{C}$ is a $C^{1}$ function then $\bar{\partial}_{M} u$ is the unique $(0,1)$-form on $M$ coinciding with $d u$ on $T_{0,1}(M)$. Similar notions may be produced for the case of a contact CR submanifold $(N, \mathscr{D})$ of a Sasakian manifold. Of course, the basic difference (with respect to CR manifolds of hypersurface type) is that we deal with the higher $\mathrm{CR}$ codimension by using a fixed complement to the invariant distribution. As before, $\omega \in \Omega^{1}(N)$ is a $(0,1)$-form if $\left.T_{1,0}(N)\right\rfloor \omega=0$ and (with the notations of Theorem 1) $\left.H(N)^{\perp}\right\rfloor \omega=0$. Then the tangential CauchyRiemann operator $\bar{\partial}_{N}: C^{\infty}(N) \rightarrow \Omega^{0,1}(N)$ is given by $\left(\bar{\partial}_{N} u\right) \bar{Z}=\bar{Z}(u)$, for every $Z \in T_{1,0}(N)$. Note that $\pi: N \rightarrow M$ is a CR map. Indeed, by (3.10)

$$
T_{1,0}(M)^{\uparrow}=\left\{X^{\uparrow}-i J X^{\uparrow}: X \in H(M)\right\}=T_{1,0}(N)
$$

where $X^{\uparrow}:=\left(d_{x} \pi: \mathscr{H}_{x} \rightarrow T_{\pi(x)}(M)\right)^{-1} X$. Then

$$
\begin{aligned}
\bar{\partial}_{N}(u \circ \pi)_{x} \bar{Z}_{x} & =\bar{Z}_{x}(u \circ \pi)=\left[\left(d_{x} \pi\right) \bar{Z}_{x}\right](u) \\
& =\left(\bar{\partial}_{M} u\right)_{\pi(x)}\left(d_{x} \pi\right) \bar{Z}_{x}=\left(\pi^{*} \bar{\partial}_{M} u\right)_{x} \bar{Z}_{x},
\end{aligned}
$$

for any $u \in C^{\infty}(M), x \in N$ and $Z \in \Gamma^{\infty}\left(T_{1,0}(N)\right)$. Next

$$
\begin{aligned}
T(N) & =\mathscr{H} \oplus \operatorname{Ker}(d \pi)=T(M)^{\uparrow} \oplus \mathbf{R} \xi \\
& =H(M)^{\uparrow} \oplus\left[H(M)^{\perp}\right]^{\uparrow} \oplus \mathbf{R} \xi=H(N) \oplus\left[H(M)^{\perp}\right]^{\uparrow} \oplus \mathbf{R} \xi,
\end{aligned}
$$

yields

$$
H(N)^{\perp}=\left[H(M)^{\perp}\right]^{\uparrow} \oplus \mathbf{R} \xi
$$

by (3.10) and the fact that the horizontal lift is a field of linear isometries (i.e. $G(X, Y)^{v}=g\left(X^{\uparrow}, Y^{\uparrow}\right)$ ). Then (by (3.13))

$$
\left.H(N)^{\perp}\right\rfloor \pi^{*} \bar{\partial}_{M} u=\left(\bar{\partial}_{M} u\right)(d \pi) H(N)^{\perp}=\left(\bar{\partial}_{M} u\right) H(M)^{\perp}=0
$$

and (3.11) is proved. The proof of (3.12) is similar. If $\left(z^{1}, \ldots, z^{n+k-1}\right)$ are local complex coordinates in $\mathbf{C P}^{n+k-1}$ then $\bar{\partial} v=Z_{\bar{j}}(v) d \bar{z}^{j}$, where $Z_{\bar{j}}=\partial / \partial \bar{z}^{j}$ and $v \in$ $C^{1}\left(\mathbf{C P}^{n+k-1}\right)$. The projection $\tilde{\pi}_{0}: \mathbf{C}^{n+k} \backslash\{0\} \rightarrow \mathbf{C P}^{n+k-1}$ is holomorphic hence $\pi_{0}: S^{2(n+k)-1} \rightarrow \mathbf{C P}^{n+k-1}$ is a CR map. Then

$$
\bar{\partial}_{S^{2(n+k)-1}}\left(v \circ \pi_{0}\right) \bar{Z}=\bar{Z}\left(v \circ \pi_{0}\right)=\left[\left(d \pi_{0}\right) \bar{Z}\right](v)=\bar{Z}\left(\pi^{\bar{j}}\right) Z_{\bar{j}}(v)
$$

where $\pi^{\bar{j}}:=\bar{z}^{j} \circ \pi_{0}$. Finally $\bar{\partial}_{S^{2(n+k)-1}}\left(v \circ \pi_{0}\right)=Z_{\bar{j}}(v)\left(\pi_{0}^{*} d \bar{z}^{j}\right)=\pi_{0}^{*} \bar{\partial} v$ and Theorem 2 is completely proved. 
R. O. Wells underlines (cf. [26]) the importance of the genericity property in the theory of the functions on a real submanifold of a complex manifold, and that there are some topological obstructions in order to have this property. Indeed, he proves (by essentially using the fact that the Euler-Poincare characteristic $\chi(M)$ of a compact submanifold $M$ is got by evaluating the Euler class of the normal bundle at the fundamental class of $M$ ) that a necessary condition in order to have genericity (for a compact orientable $m$-dimensional submanifold $M$, in a compact complex manifold $V$, homologous to zero in $H_{m}(V, \mathbf{Z})$ ) is that $\chi(M)=0$. In the case of a contact CR submanifold $N$ of a Sasakian manifold ( $N$ not necessarily tangent to the contact vector field) the genericity condition implies again $\chi(N)=0$ (cf. Theorem 3 ). The proof does not require algebraic topology technics.

Let us prove Theorem 3. Let $(N, \mathscr{D})$ be a contact CR submanifold of a Sasakian manifold $\left(N_{0},\left(\phi, \xi_{0}, \eta_{0}, g_{0}\right)\right)$, under the assumptions of Theorem 3. As $N$ is generic it follows that $N_{0}$ must be $(p+2 q)$-dimensional, where $p=\operatorname{dim}_{\mathbf{R}} \mathscr{D}_{x}$ and $q=\operatorname{dim}_{\mathbf{R}} \mathscr{D}_{x}^{\perp}, x \in N$. Assume that $\xi_{x_{0}}=0$, for some $x_{0} \in N$. Then $\mathscr{D}_{x_{0}} \subseteq$ $H\left(N_{0}\right)_{x_{0}}=\operatorname{Ker}\left(\eta_{0}\right)_{x_{0}}$ (indeed, if $v \in \mathscr{D}_{x_{0}}$ then $g_{0, x_{0}}\left(v, \xi_{0, x_{0}}\right)=g_{x_{0}}\left(v, \xi_{x_{0}}\right)=0$ ), hence $\phi_{x_{0}}$ descends to a complex structure on $\mathscr{D}_{x_{0}}$ so that $p$ must be even. Then $N_{0}$ is even dimensional, a contradiction. Therefore $\xi$ is a smooth vector field on $N$, without zeros, so that $\chi(N)=0$. Q.e.d.

\section{Canonical connections on real orientable hypersurfaces of Sasakian manifolds}

\subsection{The Levi form}

Let $\left(M, T_{1,0}(M)\right)$ be a CR manifold of arbitrary type $(n, k)$. The Levi form of $M$ is

$$
\begin{aligned}
L_{x}: T_{1,0}(M)_{x} \times T_{1,0}(M)_{x} & \rightarrow\left[T_{x}(M) \otimes_{\mathbf{R}} \mathbf{C}\right] /\left[H(M)_{x} \otimes_{\mathbf{R}} \mathbf{C}\right], \\
L_{x}(v, w) & :=i \pi_{x}\left([V, \bar{W}]_{x}\right),
\end{aligned}
$$

for $v, w \in T_{1,0}(M)_{x}, x \in M$, where $V, W \in \Gamma^{\infty}\left(T_{1,0}(M)\right)$ are chosen such that $V_{x}=v, W_{x}=w$ and

$$
\pi: T(M) \otimes \mathbf{C} \rightarrow[T(M) \otimes \mathbf{C}] /[H(M) \otimes \mathbf{C}]
$$

is the projection. Then $\left(M, T_{1,0}(M)\right)$ is nondegenerate if $L$ is nondegenerate. Let us consider the conormal bundle

$$
H(M)_{x}^{\perp}:=\left\{\omega \in T_{x}^{*}(M): \operatorname{Ker}(\omega) \supseteq H(M)_{x}\right\}, \quad x \in M .
$$

Assume $M$ to be oriented. When $k=1$, i.e. $M$ is of hypersurface type, $H(M)^{\perp}$ is an oriented line bundle, hence trivial. Thus $H(M)^{\perp}$ admits global nowhere zero sections $\theta \in \Gamma^{\infty}\left(H(M)^{\perp}\right)$, each of which is referred to as a pseudohermitian structure on $M$. The Levi form may be recast as

$$
L_{\theta}(V, \bar{W}):=-i(d \theta)(V, \bar{W}), \quad V, W \in T_{1,0}(M),
$$


and it is easily seen that $L$ and $L_{\theta}$ coincide up to a bundle isomorphism $[T(M) \otimes \mathbf{C}] /[H(M) \otimes \mathbf{C}] \approx H(M)^{\perp}$. If $L_{\theta}$ is nondegenerate then $\theta$ is a contact form, i.e. $\theta \wedge(d \theta)^{n}$ is a volume form on $M$.

If $M$ is a CR manifold of type $(n, k)$ and $\mathrm{CR}$ codimension $k \geq 2$, the conormal bundle $H(M)^{\perp}$ has rank $k$ and, in general, the orientability assumption doesn't guarantee triviality (the topological obstructions towards $H(M)^{\perp} \approx$ $M \times \mathbf{R}^{k}$ were not investigated). Given a real orientable hypersurface $N$ in a Sasakian manifold $N_{0}$ (by our Theorem 9) $N$ is a CR manifold. Let $L$ be its Levi form. $\quad N$ has CR codimension $k=2$, hence $L$ may not be computed in terms of a pseudohermitian structure. However the conormal bundle $H(N)^{\perp}$ admits the global frame $\{\eta, u\}$, where $\eta=i^{*} \eta_{0}$ and $u(X):=g(X, U), X \in T(N)$, hence $H(N)^{\perp} \approx N \times \mathbf{R}^{2}$. We shall need the bundle isomorphism

$$
\begin{gathered}
\Phi:[T(N) \otimes \mathbf{C}] /[H(N) \otimes \mathbf{C}] \rightarrow \mathbf{R} U \oplus \mathbf{R} \xi, \\
\pi_{x}(v) \mapsto \eta_{x}(v) \xi_{x}+u_{x}(v) U_{x}, \quad v \in T_{x}(N) \otimes_{\mathbf{R}} \mathbf{C}, \quad x \in N .
\end{gathered}
$$

Then $H(N)\rfloor \eta=0$ and $H(N)\rfloor u=0$ yield

$$
\Phi_{x}\left(L_{x}(v, w)\right)=-2 i\left\{(d \eta)(V, \bar{W})_{x} \xi_{x}+(d u)(V, \bar{W})_{x} U_{x}\right\} .
$$

Next, as $N_{0}$ satisfies the contact condition

$$
(d \eta)(X, Y)=g(X, \mathrm{P} Y)
$$

for any $X, Y \in T(N)$. Also

$$
\begin{gathered}
2(d u)(X, Y)=\left(\nabla_{X} u\right) Y-\left(\nabla_{Y} u\right) X, \\
\left(\nabla_{X} u\right) Y=g_{0}\left(\nabla_{X}^{0} U, Y\right) .
\end{gathered}
$$

Using the well known characterization of Sasakian structures (cf. e.g. D. E. Blair, [4], p. 73)

$$
\left(\nabla_{X}^{0} \phi\right) Y=g_{0}(X, Y) \xi-\eta_{0}(Y) X, \quad X, Y \in T\left(N_{0}\right),
$$

and the Weingarten formula, we have

$$
\begin{aligned}
\nabla_{X}^{0} U & =\nabla_{X}^{0} \phi v=\phi \nabla_{X}^{0} v+g_{0}(X, v) \xi-\eta_{0}(v) X \\
& =\phi \nabla_{X}^{0} v=\phi\left\{-a X+\nabla_{X}^{\perp} v\right\}=-\phi a X,
\end{aligned}
$$

(as $\nabla^{\perp} v=0$ ). Summing up, on any real orientable hypersurface (of a Sasakian manifold) tangent to the contact vector field

$$
\nabla_{X}^{0} U=-\phi a X
$$

so that

$$
(d u)(X, Y)=\frac{1}{2} g(X,(a \mathrm{P}+\mathrm{P} a) Y),
$$

for any $X, Y \in T(N)$. We may define

$$
\mathscr{L}(X, Y):=g(X, Y) \xi+\frac{1}{2}\{g(a X, Y)+g(a \mathrm{P} X, \mathrm{P} Y)\} U
$$


for $X, Y \in H(N)$. By the identities (4.1)-(4.2) and (4.5) it follows that

$$
\Phi_{x}\left(L_{x}(v, w)\right)=-2 \mathscr{L}(V, \bar{W})_{x}
$$

(and $\mathscr{L}$ is referred to as the Levi form of $N$, as well). Note that $\mathscr{L}$ is nondegenerate as its component along $\xi$ is precisely the first fundamental form (restricted to $H(N)$ ).

If $M$ is a CR manifold of hypersurface type, then $M$ is strictly pseudoconvex if $L_{\theta}$ is positive definite, for some pseudohermitian structure $\theta$. On the other hand, for a real orientable hypersurface $N$ in a Sasakian manifold, $\mathscr{L}$ is a vector valued form, hence there is no obvious analog to strict pseudoconvexity.

\subsection{Canonical connections}

Let us prove Theorem 4. Given a linear connection $D$ on $N$ we say its torsion $T_{D}$ is pure if

$$
T_{D}(Z, W)=0, \quad T_{D}(Z, \bar{W})=-2 i \mathscr{L}(Z, \bar{W}), \quad Z, \bar{W} \in T_{1,0}(N),
$$

where $\mathscr{L}$ is the Levi form of $N$, and

$$
\tau \circ J+J \circ \tau=0, \quad \rho \circ J+J \circ \rho=0,
$$

where $\tau, \rho$ are the vector valued 1 -forms on $N$ given by

$$
\tau X:=T_{D}(\xi, X), \quad \rho X:=T_{D}(U, \xi), \quad X \in T(N) .
$$

We establish the following more general statement.

Proposition 1. For any $\lambda \in \Omega^{1}(N)$ there is a unique linear connection $D$ on $N$ satisfying the following axioms 1) $H(N)$ is parallel which respect to $\mathscr{D}, 2)$ $D g=0$, where $g=i^{*} g_{0}$ is the first fundamental form of $\left.i: N \subset N_{0}, 3\right) D J=0$, where $J=\left.\phi\right|_{H(N)}$ is the complex structure of $\left.H(N), 4\right)$ the torsion $T_{D}$ of $D$ is pure, and 5) $D \xi=\lambda \otimes U$ and $D U=-\lambda \otimes \xi$.

Note first that $J=\phi$ along $H(N)$ and let us extend $J$ to the whole of $T(N)$ by setting $J U:=0$ and $J \xi:=0$. Then $J=\mathrm{P}$ on $T(N)$. Also, note that $T(N)=$ $H(N) \oplus \mathbf{R} U \oplus \mathbf{R} \xi$ and $T_{1,0}(N)=\{X-i \phi X: X \in H(N)\}$. At this point, we may check the uniqueness of a linear connection $D$ obeying to the axioms 1)-5) in Proposition 1. By axiom 2)

$$
X(g(Y, Z))=g\left(D_{X} Y, Z\right)+g\left(Y, D_{X} Z\right) .
$$

Let us set $Y=\xi$ and use $g(X, \xi)=\eta(X)$. Then

$$
X(\eta(Z))=g\left(D_{X} \xi, Z\right)+\eta\left(D_{X} Z\right) .
$$

Choose $Z \in H(N)$. Then (by $D_{X} Z \in H(N)$ )

$$
g\left(D_{X} \xi, Z\right)=0
$$

i.e. the component of $D_{X} \xi$ along $H(N)$ vanishes. Now we set $Z=\xi$ in (4.7) so that (by $\eta(\xi)=1$ ) 


$$
2 \eta\left(D_{X} \xi\right)=0,
$$

i.e. the component of $D_{X} \xi$ along $\xi$ vanishes. We may conclude that

$$
D_{X} \xi \in \mathbf{R} U, \quad X \in T(N) .
$$

Set $Y=U$ in (4.6). Then

$$
X(u(Z))=g\left(D_{X} U, Z\right)+u\left(D_{X} Z\right) .
$$

Next, for $Z \in H(N)$ (due to $H(N)\rfloor u=0$ )

$$
g\left(D_{X} U, Z\right)=0
$$

i.e. the component of $D_{X} U$ along $H(N)$ vanishes. Set $Z=U$ in (4.9) so that (by $u(U)=1)$

$$
2 u\left(D_{X} U\right)=0,
$$

i.e. the component of $D_{X} U$ along $U$ vanishes. Summing up

$$
D_{X} U \in \mathbf{R} \xi, \quad X \in T(N) .
$$

Let $Z, W \in T_{1,0}(N)$. By axiom 3)

$$
T_{D}(\bar{Z}, W)=2 i\{g(\bar{Z}, W) \xi+g(a \bar{Z}, W) U\},
$$

hence

$$
D_{\bar{Z}} W-D_{W} \bar{Z}-[\bar{Z}, W]=2 i\{g(\bar{Z}, W) \xi+g(a \bar{Z}, W) U\} .
$$

It follows that

$$
D_{\bar{Z}} W=[\bar{Z}, W]_{T_{1,0}(N)}, \quad D_{W} \bar{Z}=-[\bar{Z}, W]_{T_{0,1}(N)},
$$

where $V_{T_{1,0}(N)}$ and $V_{T_{0,1}(N)}$ are the projections of a $V \in T(N) \otimes \mathbf{C}$ on $T_{1,0}(N)$ and $T_{0,1}(N)$, respectively. If $V \in T_{1,0}(N)$

$$
g\left(D_{Z} W, \bar{V}\right)=Z(g(W, \bar{V}))-g\left(W, D_{Z} \bar{V}\right),
$$

and by (4.11)

$$
g\left(D_{Z} W, \bar{V}\right)=Z(g(W, \bar{V}))+g\left(W,[\bar{V}, Z]_{T_{0,1}(N)}\right) .
$$

Set $\lambda(X):=u\left(D_{X} \xi\right)$. As $g(\xi, U)=0$ and $u\left(D_{X} \xi\right)=-\eta\left(D_{X} U\right)$ we get (by (4.8) and (4.10))

$$
D_{X} \xi=\lambda(X) U, \quad D_{X} U=-\lambda(X) \xi,
$$

for any $X \in T(N)$. Next, for $X \in T(N)$

$$
\tau X=D_{\xi} X-\lambda(X) U-[\xi, X] .
$$

Applying $J$ to (4.14) we have

$$
J \tau X=J D_{\xi} X-J[\xi, X],
$$

and (replacing $X$ by $J X$ )

$$
\tau J X=D_{\xi} J X-\lambda(J X) U-[\xi, J X] .
$$


Adding up the last two identities and using $\tau \circ J+J \circ \tau=0$ and $D J=0$

$$
0=2 J D_{\xi} X-J \mathscr{L}_{\xi} X-\mathscr{L}_{\xi} J X-\lambda(J X) U,
$$

where $\mathscr{L}_{X}$ denotes the Lie derivative in the direction $X$. If $X \in H(N)$

$$
2 D_{\xi} X=-J^{2} \mathscr{L}_{\xi} X-J \mathscr{L}_{\xi} J X, \quad X \in H(N) .
$$

Indeed, for any $X \in T(N)$

$$
X=X_{H}+\eta(X) \xi+u(X) U
$$

for some $X_{H} \in H(N)$. We have $J X=J X_{H}=\phi X_{H}$ so that $J^{2} X=\phi^{2} X_{H}=-X_{H}$. Therefore

$$
J^{2} X=-X+\eta(X) \xi+u(X) U .
$$

Let us now observe that (by (4.2))

$$
\eta\left(\mathscr{L}_{\xi} X\right)=\eta([\xi, X])=-2(d \eta)(\xi, X)=-2 g(\xi, \mathrm{P} X)=0,
$$

i.e. $\mathscr{L}_{\xi} X \in H(N) \oplus \mathbf{R} U$. Moreover (by (4.5)) for $X \in H(N)$ and $Y=\xi$ one has $2(d u)(X, \xi)=g(X, \mathrm{P} a \xi)$ so that

$$
u([\xi, X])=-2(d u)(\xi, X)=g(X, \mathrm{P} a \xi)
$$

i.e. $u\left(\mathscr{L}_{\xi} X\right)=g(\mathrm{P} a \xi, X)$. Then

$$
J^{2} \mathscr{L}_{\xi} X=-\mathscr{L}_{\xi} X+g(\mathrm{P} a \xi, X) U
$$

which together with (4.15) leads to

$$
2 D_{\xi} X=\mathscr{L}_{\xi} X-g(\mathrm{P} a \xi, X) U-J \mathscr{L}_{\xi} J X .
$$

Note that for $X \in H(N)$ one has $\left(\mathscr{L}_{\xi} J\right) J X=-\mathscr{L}_{\xi} X-J \mathscr{L}_{\xi} J X$. Then our last identity may be also written

$$
D_{\xi} X=\mathscr{L}_{\xi} X+\frac{1}{2}\left(\mathscr{L}_{\xi} J\right) J X-\frac{1}{2} g(\mathrm{P} a \xi, X) U,
$$

for any $X \in H(N)$. On the other hand, by the Gauss equation of $N$ in $N_{0}$ and using $\nabla_{V}^{0} \xi=-\phi V$ (for any $V \in T\left(N_{0}\right)$ ) we obtain

$$
\nabla_{X} \xi=-\mathrm{P} X, \quad g(a X, \xi)=-\mathrm{F} X .
$$

Yet

$$
\mathrm{F} X=-u(X) v
$$

so that

$$
\nabla_{X} \xi=-\mathrm{P} X, \quad \eta(a X)=u(X) .
$$

Let us observe that

$$
\left\{\begin{array}{l}
a X \in H(N) \oplus \mathbf{R} U, \quad X \in H(N) \\
a U=\xi+Y, \quad Y \in H(N) \oplus \mathbf{R} U \\
a \xi \in H(N) \oplus \mathbf{R} U
\end{array}\right.
$$


Then $g(P a \xi, X)=0$. Consider

$$
T_{\xi}: H(N) \rightarrow H(N), \quad T_{\xi}:=\frac{1}{2}\left(\mathscr{L}_{\xi} J\right) J X, \quad X \in H(N) .
$$

The identity (4.16) becomes

$$
D_{\xi} X=\mathscr{L}_{\xi} X+T_{\xi} X,
$$

for any $X \in H(N)$. For $X \in T(N)$ (by (4.13))

$$
\rho X=D_{U} X+\lambda(X) \xi-[U, X] .
$$

By applying $J$ to $(4.20)$ we obtain

$$
J \rho X=J D_{U} X-J[U, X],
$$

hence (replacing $X$ by $J X$ )

$$
\rho J X=D_{U} J X+\lambda(J X) \xi-[U, J X] .
$$

Adding up the last two identities and using $\rho \circ J+J \circ \rho=0$ and $D J=0$ we obtain

$$
0=2 J D_{U} X-J \mathscr{L}_{U} X-\mathscr{L}_{U} J X+\lambda(J X) \xi .
$$

If $X \in H(N)($ as $H(N)$ is $D$-parallel)

$$
2 D_{U} X=-J^{2} \mathscr{L}_{U} X-J \mathscr{L}_{U} J X, \quad X \in H(N) .
$$

Now (by (4.2))

$$
\eta\left(\mathscr{L}_{U} X\right)=\eta([U, X])=-2(d \eta)(U, X)=-2 g(U, \mathbf{P} X)=0,
$$

i.e. $\mathscr{L}_{U} X \in H(N) \oplus \mathbf{R} U$. Moreover (by (4.5))

$$
u\left(\mathscr{L}_{U} X\right)=u([U, X])=-2(d u)(U, X)=-g(U,(a \mathrm{P}+\mathrm{P} a) X),
$$

i.e. $u\left(\mathscr{L}_{U} X\right)=-u(a \mathrm{P} X) \quad($ as $\mathrm{P} U=\tan \{\phi U\}=-\tan \{v\}=0)$. Then

$$
J^{2} \mathscr{L}_{U} X=-\mathscr{L}_{U} X-u(a \mathrm{P} X) U,
$$

which together with (4.21) leads to

$$
2 D_{U} X=\mathscr{L}_{U} X-J \mathscr{L}_{U} J X+u(a \mathrm{P} X) U .
$$

Note that for $X \in H(N)$ one has $\left(\mathscr{L}_{U} J\right) J X=-\mathscr{L}_{U} X-J \mathscr{L}_{U} J X$. Let us consider

$$
T_{U}: H(N) \rightarrow H(N) \oplus \mathbf{R} U, \quad T_{U} X:=\frac{1}{2}\left(\mathscr{L}_{U} J\right) J X, \quad X \in H(N) .
$$

Then

$$
D_{U} X=\mathscr{L}_{U} X+T_{U} X+\frac{1}{2} u(a \mathrm{P} X) U .
$$


Uniqueness is proved. We proceed by proving the existence of a connection $D$ obeying to 1)-5). To this end, we consider the differential operator

$$
D_{X}: \Gamma^{\infty}(T(N)) \rightarrow \Gamma^{\infty}(T(N)), \quad X \in \mathscr{X}(N),
$$

defined by (4.11), (4.12), (4.13), (4.19) and (4.22), for a fixed $\lambda \in \Omega^{1}(N)$. It may be easily checked that $D$ is a linear connection on $N$. The only property requesting a bit of care is $D_{X} f Y=X(f) Y+f D_{X} Y$. Let $f \in C^{\infty}(N)$. Then for any $Z, W \in T_{1,0}(N)$

$$
D_{f \bar{Z}} W=[f \bar{Z}, W]_{T_{1,0}(N)}=(f[\bar{Z}, W]-W(f) \bar{Z})_{T_{1,0}(N)}=f D_{\bar{Z}} W
$$

(as $\left.\bar{Z}_{T_{1,0}(N)}=0\right)$. Also

$$
\begin{gathered}
D_{\xi}(f X)=T_{\xi}(f X)+\mathscr{L}_{\xi}(f X)=f D_{\xi} X+\xi(f) X, \\
D_{U}(f X)=T_{U}(f X)+\mathscr{L}_{U}(f X)+\frac{1}{2} u(a \mathrm{P}(f X))=f D_{U} X+U(f) X,
\end{gathered}
$$

etc.

\subsection{The torsions $\tau$ and $\rho$}

We establish a few useful properties of $\tau$ and $\rho$, when $\lambda \equiv 0$ (from now $D$ is the connection furnished by Theorem 4). Let $X \in T(N)$ and $Z \in H(N)$. By (4.3)

$$
\left(\nabla_{X}^{0} \phi\right) Z=g(X, Z) \xi
$$

By the Gauss formula (of $N$ in $N_{0}$ )

$$
\begin{aligned}
g(X, Z) \xi & =\left(\nabla_{X}^{0} \phi\right) Z=\nabla_{X}^{0} \mathrm{P} Z-\phi \nabla_{X}^{0} Z \\
& =\nabla_{X} \mathrm{P} Z+g(a X, \mathrm{P} Z) v-\phi\left\{\nabla_{X} Z+g(a X, Z) v\right\} \\
& =\nabla_{X} \mathrm{P} Z+g(a X, \mathrm{P} Z) v-\mathrm{P}_{X} Z-\mathrm{F}_{X} Z-g(a X, Z) U,
\end{aligned}
$$

where from (by identifying the tangential components)

$$
\nabla_{X} \mathrm{P} Z=\mathrm{P} \nabla_{X} Z+g(X, Z) \xi+g(a X, Z) U,
$$

for any $X \in T(N), Z \in H(N)$, and (by identifying the normal components)

$$
g(a X, \mathrm{P} Z) v-\mathrm{F}_{X} Z=0 .
$$

Next (by $\mathrm{F} X=-u(X) v$, for any $X \in T(N)$ ) we obtain

$$
g(a X, \mathrm{P} Z)+u\left(\nabla_{X} Z\right)=0,
$$

for any $X \in T(N), Z \in H(N)$.

Let $X \in H(N)$. By (4.19) and (4.22)

$$
\tau X=T_{\xi} X, \quad \rho X=T_{U} X+\frac{1}{2} u(a \mathrm{P} X) U .
$$

On the other hand $T_{D}(\xi, U)=-[\xi, U]$ so that

$$
\tau U=-\rho \xi=-[\xi, U] .
$$


By (4.5)

$$
u([\xi, U])=-2(d u)(\xi, U)=-g(\xi,(a \mathrm{P}+\mathrm{P} a) U)=0,
$$

while by $(4.2)$

$$
\eta([\xi, U])=-2(d \eta)(\xi, U)=-2 g(\xi, \mathrm{P} U)=0 .
$$

Finally, for $Y \in H(N)$ (by using (4.4) and (4.18))

$$
g([\xi, U], Y)=g\left(\nabla_{\xi} U, Y\right)-g\left(\nabla_{U} \xi, Y\right)=-g(\phi a \xi, Y)+g(\mathrm{P} U, X)=0,
$$

i.e. the component of $[\xi, U]$ along $H(N)$ vanishes. Then $[\xi, U]=0$ so that $\tau U=\rho \xi=0$. Summing up, the vector valued 1-forms $\tau$ and $\rho$ satisfy

$$
\begin{gathered}
\left\{\begin{array}{l}
\tau X=T_{\xi} X, \quad X \in H(N), \\
\tau \xi=\tau U=0
\end{array}\right. \\
\left\{\begin{array}{l}
\rho X=T_{U} X+\frac{1}{2} u(a \mathrm{P} X) U, \quad X \in H(N) . \\
\rho \xi=\rho U=0
\end{array}\right.
\end{gathered}
$$

We also establish

Lemma 2. For every $X, Y \in H(N)$

$$
g\left(T_{\xi} X, Y\right)=g\left(X, T_{\xi} Y\right), \quad g\left(T_{U} X, Y\right)=g\left(X, T_{U} Y\right) .
$$

The proof is a straightforward calculation based on (4.4), (4.18) and (4.23). Using $g(u(a \mathrm{P} X) U, Y)=0$, for any $X, Y \in H(N)$, and the previous lemma we obtain

COROLlaRY 1. $\tau, \rho$ are self adjoint along the maximally complex distribution, i.e.

$$
g(\tau X, Y)=g(X, \tau Y), \quad g(\rho X, Y)=g(X, \rho Y) .
$$

for any $X, Y \in H(N)$.

Note that $\eta\left(\mathscr{L}_{\xi} X\right)=0, u\left(\mathscr{L}_{\xi} X\right)=0$, for any $X \in H(N)$, i.e. $\mathscr{L}_{\xi} H(N) \subseteq H(N)$ so that $\tau H(N) \subseteq H(N)$. On the other hand (by $\eta\left(\mathscr{L}_{U} X\right)=0$ and $u\left(\mathscr{L}_{U} X\right)=$ $-u(a \mathrm{P} X)$, for any $X \in H(N))$ one has $u(\rho X)=u(a \mathrm{P} X)$ and then $u\left(T_{U} X\right)=$ $\frac{1}{2} u(a \mathrm{P} X)$, for $X \in H(N)$, i.e. $\rho H(N) \subseteq H(N) \oplus \mathbf{R} U$. Moreover, if $X, Y \in T(N)$, by the uniqueness of the direct sum decomposition $T(N)=H(N) \oplus \mathbf{R} U \oplus \mathbf{R} \xi$

$$
\begin{aligned}
g(\rho X, Y) & =g\left(\rho X_{H}, Y\right) \\
& =g\left(\rho X_{H}, Y_{H}\right)+\eta(Y) g\left(\rho X_{H}, \xi\right)+u(Y) g\left(\rho X_{H}, U\right) \\
& =g\left(X_{H}, \rho Y_{H}\right)+u(Y) u\left(a \mathrm{P} X_{H}\right) \\
& =g\left(X, \rho Y_{H}\right)-\eta(X) g\left(\xi, \rho Y_{H}\right)-u(X) g\left(U, \rho Y_{H}\right)+u(Y) u\left(a \mathrm{P} X_{H}\right)
\end{aligned}
$$

where $X_{H}$ is the $H(N)$-component of $X$. Summing up 
COROLlary 2. For any $X, Y \in T(N)$

$$
g(\rho X, Y)=g(X, \rho Y)-u(X) u(a \mathrm{P} Y)+u(Y) u(a \mathrm{P} X)
$$

and

$$
g(\tau X, Y)=g(X, \tau Y)
$$

i.e. $\tau$ is self-adjoint [as an endomorphism of $(T(N), g)]$.

We proceed by deriving an useful relation between the canonical connection $D$ and the Levi-Civita connection $\nabla$ of $N$. As both $D$ and $\nabla$ are metric connections

$$
\begin{aligned}
2 g\left(D_{X} Y, Z\right)= & 2 g\left(\nabla_{X} Y, Z\right)+g\left(T_{D}(X, Y), Z\right) \\
& +g\left(T_{D}(Z, X), Y\right)+g\left(T_{D}(Z, Y), X\right)
\end{aligned}
$$

holds for any $X, Y, Z \in T(N)$. By the purity axiom

$$
T_{D}(X, Y)=2 g(X, \mathrm{P} Y) \xi-\{g(a \mathrm{P} X, Y)-g(a X, \mathrm{P} Y)\} U,
$$

for any $X, Y \in H(N)$. Also, for $Z \in H(N)$ and $X, Y \in T(N)$

$$
T_{D}(Z, X)=T_{D}\left(Z, X_{H}\right)+\eta(X) T_{D}(Z, \xi)+u(X) T_{D}(Z, U),
$$

where from

$$
T_{D}(Z, X)=2 g\left(Z, \mathrm{P} X_{H}\right) \xi-\left\{g\left(a \mathrm{P} Z, X_{H}\right)-g\left(a Z, \mathrm{P} X_{H}\right)\right\} U-\eta(X) \tau Z-u(X) \rho Z .
$$

The torsion expression together with (4.29) leads to

$$
\begin{aligned}
2 g\left(D_{X} Y, Z\right)= & 2 g\left(\nabla_{X} Y, Z\right)+g\left(T_{D}(X, Y), Z\right) \\
& +2 g\left(Z, \mathrm{P} X_{H}\right) \eta(Y)+2 g\left(Z, \mathrm{P} Y_{H}\right) \eta(X) \\
& -\left\{g\left(a \mathrm{P} Z, X_{H}\right)-g\left(a Z, \mathrm{P} X_{H}\right)\right\} u(Y) \\
& -\left\{g\left(a \mathrm{P} Z, Y_{H}\right)-g\left(a Z, \mathrm{P} Y_{H}\right)\right\} u(X) \\
& -\eta(X) g(\tau Z, Y)-u(X) g(\rho Z, Y) \\
& -\eta(Y) g(\tau Z, X)-u(Y) g(\rho Z, X),
\end{aligned}
$$

for any $X, Y \in T(N), Z \in H(N)$. If $Y \in H(N)$ then (by $D_{X} Y \in H(N)$ )

$$
\begin{aligned}
2 D_{X} Y= & 2\left(\nabla_{X} Y\right)_{H}+\left(T_{D}(X, Y)\right)_{H}+u(X)\{(a \mathrm{P}+\mathrm{P} a) Y\}_{H} \\
& +2 \eta(X) \mathrm{P} Y-\eta(X) \tau Y-u(X)(\rho Y)_{H} .
\end{aligned}
$$

Now, since $X=X_{H}+\eta(X) \xi+u(X) U \in T(N)$

$$
\left(T_{D}(X, Y)\right)_{H}=\left\{T_{D}\left(X_{H}, Y\right)+\eta(X) \tau Y+u(X) \rho Y\right\}_{H}
$$

and (by $\left.T_{D}\left(X_{H}, Y\right) \in \mathbf{R} U \oplus \mathbf{R} \xi\right)$

$$
\left(T_{D}(X, Y)\right)_{H}=\eta(X) \tau Y+u(X)(\rho Y)_{H},
$$


where from

$$
2 D_{X} Y=2\left(\nabla_{X} Y\right)_{H}+u(X)\{(a \mathrm{P}+\mathrm{P} a) Y\}_{H}+2 \eta(X) \mathrm{P} Y .
$$

On the other hand, due to $u((a \mathrm{P}+\mathrm{P} a) Y)=u(a \mathrm{P} Y)$ and $\eta((a \mathrm{P}+\mathrm{P} a) Y)=0$

$$
\{(a \mathrm{P}+\mathrm{P} a) Y\}_{H}=(a \mathrm{P}+\mathrm{P} a) Y-u(a \mathrm{P} Y) U
$$

Moreover (by (4.23))

$$
\eta\left(\nabla_{X} Y\right)=X(g(Y, \xi))-g\left(Y, \nabla_{X} \xi\right)=-g(X, \mathrm{P} Y)
$$

and (by (4.2))

$$
u\left(\nabla_{X} Y\right)=X(g(Y, U))-g\left(Y, \nabla_{X} U\right)=-g(a X, \mathrm{P} Y)
$$

so that

$$
\left(\nabla_{X} Y\right)_{H}=\nabla_{X} Y+g(X, \mathrm{P} Y) \xi+g(a X, \mathrm{P} Y) U .
$$

Let us substitute in (4.33) so that to obtain the identity

$$
\begin{aligned}
D_{X} Y= & \nabla_{X} Y+g(X, \mathrm{P} Y) \xi+\frac{1}{2}\{2 g(a X, \mathrm{P} Y)-u(X) u(a \mathrm{P} Y)\} U \\
& +\frac{1}{2}\{2 \eta(X) \mathrm{P} Y+u(X)(a \mathrm{P}+\mathrm{P} a) Y\},
\end{aligned}
$$

for any $X \in T(N), Y \in H(N)$. When $X \in H(N)$ (respectively $X=U$, or $X=\xi$ ) we obtain

$$
\begin{gathered}
D_{X} Y=\nabla_{X} Y+g(X, \mathrm{P} Y) \xi+g(a X, \mathrm{P} Y) U, \\
D_{U} Y=\nabla_{U} Y+\frac{1}{2}\{u(a \mathrm{P} Y) U+(a \mathrm{P}+\mathrm{P} a) Y\}, \\
D_{\xi} Y=\nabla_{\xi} Y+\mathrm{P} Y,
\end{gathered}
$$

for any $X, Y \in H(N)$.

\section{Applications}

\subsection{A curvature formula}

Let us consider a $\mathrm{C}-\mathrm{R}$ fibration over a real orientable hypersurface $M \subset \mathbf{C P}^{n}$, i.e.

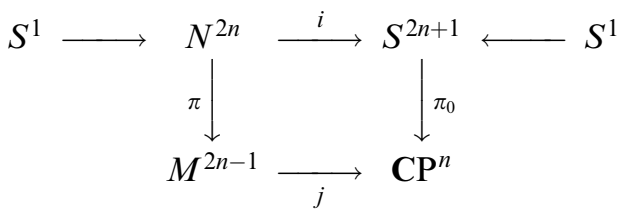

under the further assumption that the natural almost contact structure of $M$ (as real hypersurface of $\mathbf{C P}^{n}$ ) satisfies the contact condition $d \eta_{M}=\Omega_{M}$, where 
$\eta_{M}(X):=G\left(X, J_{\mathrm{CP}^{n}} v_{M}\right)$ is the contact form, $v_{M}$ is a (globally defined) unit normal field on $M$, and $J_{\mathrm{CP}^{n}}$ is the complex structure of $\mathbf{C P}^{n}$. Also, we set $\Omega_{M}(X, Y)=G\left(X, \mathrm{P}_{M} Y\right)$, for $X, Y \in T(M)$. In general, if $\left(M, T_{1,0}(M)\right)$ is a CR manifold of hypersurface type and if $\theta \in \Gamma^{\infty}\left(H(M)^{\perp}\right)$ is a pseudohermitian structure, then the real Levi form is

$$
G_{\theta}(X, Y):=(d \theta)(X, J Y), \quad X, Y \in H(M) .
$$

As $G_{\theta}(J X, J Y)=G_{\theta}(X, Y)$ (as consequence of the formal integrability of $\left.T_{1,0}(M)\right)$ it follows that $L_{\theta}$ and the (C-linear) extension of $G_{\theta}$ actually coincide (on $T_{1,0}(M) \otimes T_{0,1}(M)$ ). If $M$ is nondegenerate there is (cf. e.g. S. Dragomir, [9]) a globally defined nowhere zero vector field $T \in \mathscr{X}(M)$, transverse to the Levi distribution $H(M)$, uniquely determined by $\theta(T)=1, T\rfloor d \theta=0 . \quad T$ is the characteristic direction of $d \theta$. The Webster metric $g_{\theta}$ is

$$
\begin{gathered}
g_{\theta}=G_{\theta} \quad \text { on } H(M) \otimes H(M), \\
g_{\theta}(X, T)=\theta(X), \quad X \in T(M) .
\end{gathered}
$$

$g_{\theta}$ is a semi-Riemannian metric on $M$ (of signature $(2 r+1,2 s)$, where $(r, s)$ is the signature of the Levi form $\left.L_{\theta}\right)$. If $M$ is strictly pseudoconvex and $L_{\theta}$ is positive definite, then $g_{\theta}$ is a Riemannian metric on $M$ and $\left(J, T, \theta, g_{\theta}\right)$ is a contact metric structure (which is normal if and only if the Tanaka-Webster connection of $(M, \theta)$ has a vanishing pseudohermitian torsion, cf. [9]). For the given C-R fibration $S^{1} \rightarrow N \rightarrow M$ the 1 -form $\theta:=-\eta_{M}$ is a pseudohermitian structure on $M$. Moreover, for $X, Y \in H(M)$

$$
G_{\theta}(X, Y)=(d \theta)\left(X, J_{M} Y\right)=-\left(d \eta_{M}\right)\left(X, J_{M} Y\right)=-\Omega_{M}\left(X, J_{M} Y\right),
$$

hence $G_{\theta}=G$ on $H(M) \otimes H(M)$. In particular $M$ is strictly pseudoconvex. Let $T$ the characteristic direction of $d \theta$. In general, given an a.ct.m. structure $(\phi, \xi, \eta, g)$, the underlying contact field is not characteristic for $d \eta$. However, in the case at hand, if $M$ satisfies the contact condition then

LEMMA 3.

$$
T=-J_{\mathbf{C P}^{n}} v_{M} .
$$

Proof. Let $U_{M}:=J_{\mathrm{CP}^{n}} v_{M} \in \mathscr{X}(M)$. Then

$$
\begin{aligned}
& \theta\left(-U_{M}\right)=\eta_{M}\left(U_{M}\right)=G\left(U_{M}, U_{M}\right)=1, \\
& \left.\left.\left.-U_{M}\right\rfloor d \theta=U_{M}\right\rfloor d \eta_{M}=U_{M}\right\rfloor \Omega_{M}=0,
\end{aligned}
$$

because $\mathrm{P}_{M} U_{M}=0$. Then $T=-U_{M}$. Q.e.d.

We also have $g_{\theta}(T, T)=1, G(T, T)=G\left(U_{M}, U_{M}\right)=1$ and $g_{\theta}(X, T)=0=$ $G(X, T)$, for any $X \in H(M)$. Then, as $T=-U_{M}=J_{\mathrm{CP}^{n}} v_{M}$ and $\theta=-\eta_{M}$, it follows that $g_{\theta}=G$, hence the Levi-Civita connection of the Webster metric and the induced connection actually coincide. For the remainder of this paper, we 
adopt the following notations: $D$ is the canonical connection given by Theorem 4, $\nabla$ and $\nabla^{M}$ are respectively the Levi-Civita connection of $N$ and $M$, and $D^{M}$ is the Tanaka-Webster connection of $M$. Summing up, these connections are tied by the following identities

$$
\begin{gathered}
D_{X} Y=\nabla_{X} Y+g(X, \mathrm{P} Y) \xi+\frac{1}{2}\{2 g(a X, \mathrm{P} Y)-u(X) u(a \mathrm{P} Y)\} U \\
+\frac{1}{2}\{2 \eta(X) \mathrm{P} Y+u(X)(a \mathrm{P}+\mathrm{P} a) Y\}, \\
D_{X} Y=\nabla_{X} Y+g(X, \mathrm{P} Y) \xi+g(a X, \mathrm{P} Y) U, \\
D_{U} Y=\nabla_{U} Y+\frac{1}{2}\{u(a \mathrm{P} Y) U+(a \mathrm{P}+\mathrm{P} a) Y\}, \\
D_{\xi} Y=\nabla_{\xi} Y+\mathrm{P} Y,
\end{gathered}
$$

for any $X, Y \in H(N)$, and

$$
\nabla_{X^{\dagger}} Y^{\uparrow}=\left(\nabla_{X}^{M} Y\right)^{\uparrow}-G\left(X, \mathrm{P}_{M} Y\right)^{v} \xi,
$$

for any $X, Y \in T(M)$. Moreover, we need (cf. e.g. [1])

$$
\begin{aligned}
\nabla_{X}^{M} Y= & D_{X}^{M} Y+\left\{G\left(X, \mathrm{P}_{M} Y\right)-G\left(X, \tau_{M} Y\right)\right\} T \\
& +\theta(Y) \tau_{M} X+\theta(X) \mathrm{P}_{M} Y+\theta(Y) \mathrm{P}_{M} X
\end{aligned}
$$

(relating the Levi-Civita and Tanaka-Webster connections of $M$ ) where $\tau_{M}$ (the pseudohermitian torsion) is given by $\tau_{M} X=T_{D^{M}}(T, X)$. Also, we shall make use of

$$
\begin{gathered}
\tau_{M} \circ \mathrm{P}_{M}+\mathrm{P}_{M} \circ \tau_{M}=0, \quad \tau_{M} H(M) \subseteq H(M), \\
G\left(\tau_{M} X, Y\right)=G\left(X, \tau_{M} Y\right), \quad \text { for any } X, Y \in T(M),
\end{gathered}
$$

where $T_{D^{M}}$ is the torsion of $D^{M}$. Let $v$ be a global unit normal field on $N$. Note that $\left(T(M)^{\perp}\right)^{\uparrow}=T(N)^{\perp}$ yields $v_{M}^{\uparrow}=v$, so that $T^{\uparrow}=-U=-\phi v$. Moreover (by (3.5))

$$
a X^{\uparrow}=(A X)^{\uparrow}-G(X, T)^{v} \xi
$$

where $A=A_{v_{M}}$ is the Weingarten operator of $M$ associated to the normal section $v_{M}$. In particular

$$
\begin{gathered}
a X^{\uparrow}=(A X)^{\uparrow}, \quad X \in H(M), \\
a U=-a T^{\uparrow}=-(A T)^{\uparrow}+\xi .
\end{gathered}
$$

Moreover $u\left(X^{\uparrow}\right)=-(\theta(X))^{v}$, hence

$$
\left\{\begin{array}{l}
u\left(X^{\uparrow}\right)=0, \quad X \in H(M), \\
u\left(T^{\uparrow}\right)=-1 .
\end{array}\right.
$$


Let $X \in T(M), Y \in H(M)$. Then $X^{\uparrow} \in H(N) \oplus \mathbf{R} U, Y^{\uparrow} \in H(N)$, and $\eta\left(X^{\uparrow}\right)=0$ so that (by (5.1) and (5.5)-(5.6))

$$
\begin{aligned}
D_{X^{\uparrow}} Y^{\uparrow}= & \left(D_{X}^{M} Y\right)^{\uparrow}-\frac{1}{2}\left(\theta(X) \theta\left(A \mathrm{P}_{M} Y\right)\right)^{v} U \\
& +\frac{1}{2}(\theta(X))^{v}\left\{2 \mathrm{P}_{M} Y-\left(A \mathrm{P}_{M}+\mathrm{P}_{M} A\right) Y\right\}^{\uparrow} \\
& -\left\{G\left(X, \mathrm{P}_{M} Y\right)-G\left(X, \tau_{M} Y\right)-G\left(A X, \mathrm{P}_{M} Y\right)\right\}^{v} U
\end{aligned}
$$

for any $X \in T(M), Y \in H(M)$. In particular

$$
D_{X^{\dagger}} Y^{\uparrow}=\left(D_{X}^{M} Y\right)^{\uparrow}-\left\{G\left(X, \mathrm{P}_{M} Y\right)-G\left(X, \tau_{M} Y\right)-G\left(A X, \mathrm{P}_{M} Y\right)\right\}^{v} U,
$$

and

$$
D_{U} Y^{\uparrow}=-\left(D_{T}^{M} Y\right)^{\uparrow}+\frac{1}{2}\left(\theta\left(A \mathrm{P}_{M} Y\right)\right)^{v} U-\frac{1}{2}\left\{2 \mathrm{P}_{M} Y-\left(A \mathrm{P}_{M}+\mathrm{P}_{M} A\right) Y\right\}^{\uparrow}
$$

for any $X, Y \in H(M)$. Also (by (5.4) and (4.18))

$$
D_{\xi} X^{\uparrow}=\nabla_{\xi} X^{\uparrow}+\left(\mathrm{P}_{M} X\right)^{\uparrow}=\left[\xi, X^{\uparrow}\right] \in H(N) .
$$

As $\operatorname{Ker}(d \pi)=\mathbf{R} \xi$ we have

$$
d \pi\left[\xi, X^{\uparrow}\right]=\left[d \pi(\xi), d \pi\left(X^{\uparrow}\right)\right]=0, \quad X \in T(M),
$$

and then $\left[\xi, X^{\uparrow}\right] \in(H(N) \oplus \mathbf{R} U) \cap \mathbf{R} \xi=(0)$, i.e. $\left[\xi, X^{\uparrow}\right]=0$ for any $X \in T(M)$. Thus $D_{\xi} X^{\uparrow}=0$. Note also that

$$
\tau X^{\uparrow}=D_{\xi} X^{\uparrow}-\left[\xi, X^{\uparrow}\right]=T_{\xi} X^{\uparrow}
$$

and then (by $\left.H(M)^{\uparrow}=H(N)\right)$ it follows that $T_{\xi}=0$, i.e. $\tau=0 . \quad$ If $X, Y \in T(N)$ (by $(5.5))$

$$
\left[X^{\uparrow}, Y^{\uparrow}\right]=[X, Y]^{\uparrow}-2 G\left(X, \mathrm{P}_{M} Y\right)^{v} \xi .
$$

In particular $\left[U, X^{\uparrow}\right]=-[T, X]^{\uparrow}$. Let $X, Y, Z \in H(M)$. A straightforward calculation (based on (5.8)) leads to

$$
\begin{aligned}
D_{X^{\dagger}} D_{Y^{\dagger}} Z^{\uparrow}= & \left(D_{X}^{M} D_{Y}^{M} Z\right)^{\uparrow}-\left\{G\left(X, \mathrm{P}_{M} D_{Y}^{M} Z\right)-G\left(A X, \mathrm{P}_{M} D_{Y}^{M} Z\right)\right. \\
& -G\left(X, \tau_{M} D_{Y}^{M} Z\right)+X\left(G\left(Y, \mathrm{P}_{M} Z\right)\right)-X\left(G\left(A Y, \mathrm{P}_{M} Z\right)\right) \\
& \left.-X\left(G\left(Y, \tau_{M} Z\right)\right)\right\}^{v} U .
\end{aligned}
$$

Using (5.7), $D_{\xi} Z^{\uparrow}=0$, and $[X, Y]^{\uparrow} \in H(N) \oplus \mathbf{R} U$

$$
\begin{aligned}
D_{\left[X^{\dagger}, Y^{\dagger}\right]} Z^{\uparrow}=\left(D_{[X, Y]}^{M} Z\right)^{\uparrow}-\frac{1}{2}\left(\theta([X, Y]) \theta\left(A \mathrm{P}_{M} Z\right)\right)^{v} U \\
+\frac{1}{2}(\theta([X, Y]))^{v}\left\{2 \mathrm{P}_{M} Z-\left(A \mathrm{P}_{M}+\mathrm{P}_{M} A\right) Z\right\}^{\uparrow} \\
-\left\{G\left([X, Y], \mathrm{P}_{M} Z\right)-G\left([X, Y], \tau_{M} Z\right)-G\left(A[X, Y], \mathrm{P}_{M} Z\right)\right\}^{v} U .
\end{aligned}
$$


At this point, a rather lengthy (yet once again straightforward) calculation (based on (5.10)-(5.11) and $\left.D^{M} G=0\right)$ relates the curvature tensor fields of $D$ and $D^{M}$

$$
\begin{aligned}
R^{D}\left(X^{\uparrow}, Y^{\uparrow}\right) Z^{\uparrow}=\left(R^{D^{M}}(X, Y) Z\right)^{\uparrow} & -\left\{G\left(D_{X}^{M} Y, \mathrm{P}_{M} Z\right)-G\left(D_{Y}^{M} X, \mathrm{P}_{M} Z\right)-G\left([X, Y], \mathrm{P}_{M} Z\right)\right. \\
& -G\left(D_{X}^{M} A Y, \mathrm{P}_{M} Z\right)+G\left(D_{Y}^{M} A X, \mathrm{P}_{M} Z\right)+G\left(A[X, Y], \mathrm{P}_{M} Z\right) \\
& \left.-G\left(D_{X}^{M} \tau_{M} Y, Z\right)+G\left(D_{Y}^{M} \tau_{M} X, Z\right)+G\left(\tau_{M}[X, Y], Z\right)\right\}^{v} U \\
& +\frac{1}{2}\left(\theta([X, Y]) \theta\left(A \mathrm{P}_{M} Z\right)\right)^{v} U \\
& -\frac{1}{2}(\theta([X, Y]))^{v}\left\{2 \mathrm{P}_{M} Z-\left(A \mathrm{P}_{M}+\mathrm{P}_{M} A\right) Z\right\}^{\uparrow} .
\end{aligned}
$$

Note that (by $T_{D^{M}}(X, Y) \in \mathbf{R} T$ )

$$
G\left(D_{X}^{M} Y, \mathrm{P}_{M} Z\right)-G\left(D_{Y}^{M} X, \mathrm{P}_{M} Z\right)-G\left([X, Y], \mathrm{P}_{M} Z\right)=G\left(T_{D^{M}}(X, Y), \mathrm{P}_{M} Z\right)=0 .
$$

Also

$$
\theta(A X)=G(T, A X)=-G\left(T, \nabla_{X}^{\mathrm{CP}^{n}} v_{M}\right)=G\left(T, \nabla_{X}^{\mathrm{CP}^{n}} J_{\mathrm{CP}^{n}} T\right)=0,
$$

for any $X \in H(M)$. Then (by (5.6))

$$
G\left(D_{X}^{M} A Y, \mathrm{P}_{M} Z\right)=G\left(\nabla_{X}^{M} A Y, \mathrm{P}_{M} Z\right),
$$

so that

$$
\begin{aligned}
& G\left(D_{X}^{M} A Y-D_{Y}^{M} A X-A[X, Y], \mathrm{P}_{M} Z\right) \\
& \quad=G\left(\nabla_{X}^{M} A Y-\nabla_{Y}^{M} A X-A \nabla_{X}^{M} Y+A \nabla_{Y}^{M} X, \mathrm{P}_{M} Z\right) \\
& \quad=G\left(\left(\nabla_{X}^{M} A\right) Y-\left(\nabla_{Y}^{M} A\right) X, \mathrm{P}_{M} Z\right) .
\end{aligned}
$$

On the other hand, we need to recall (cf. K. Yano \& M. Kon, [29], p. 153) the Codazzi equation of $M$ (as a real hypersurface of a complex space form of holomorphic sectional curvature 4)

$$
\left(\nabla_{X}^{M} A\right) Y-\left(\nabla_{Y}^{M} A\right) X=\theta(X) \mathrm{P}_{M} Y-\theta(Y) \mathrm{P}_{M} X+2 G\left(X, \mathrm{P}_{M} Y\right) T=2 G\left(X, \mathrm{P}_{M} Y\right) T,
$$

for any $X, Y \in H(M)$. Therefore

$$
G\left(D_{X}^{M} A Y-D_{Y}^{M} A X-A[X, Y], \mathrm{P}_{M} Z\right)=0 .
$$

Next, the identities

$$
\begin{aligned}
D_{X}^{M} \tau_{M} Y & =D_{X}^{M} D_{T}^{M} Y-D_{X}^{M}[T, Y], \\
D_{Y}^{M} \tau_{M} X & =D_{Y}^{M} D_{T}^{M} X-D_{Y}^{M}[T, X], \\
\tau_{M}[X, Y] & =D_{T}^{M}[X, Y]-[T,[X, Y]],
\end{aligned}
$$

lead to 


$$
\begin{aligned}
& G\left(D_{X}^{M} \tau_{M} Y-D_{Y}^{M} \tau_{M} X-\tau_{M}[X, Y], Z\right) \\
&= G\left(R^{D^{M}}(X, T) Y-R^{D^{M}}(Y, T) X+D_{[X, T]}^{M} Y-D_{[Y, T]}^{M} X, Z\right) \\
&+G\left(D_{X}^{M}[Y, T]-D_{Y}^{M}[X, T]+[T,[X, Y]], Z\right) \\
&= G\left(R^{D^{M}}(X, T) Y-R^{D^{M}}(Y, T) X, Z\right) .
\end{aligned}
$$

Finally (by $\left.\theta([X, Y])=-2(d \theta)(X, Y)=2 G\left(X, \mathrm{P}_{M} Y\right)\right)$ (5.12) becomes

$$
\begin{aligned}
R^{D}\left(X^{\uparrow}, Y^{\uparrow}\right) Z^{\uparrow}= & \left(R^{D^{M}}(X, Y) Z\right)^{\uparrow} \\
& +G\left(R^{D^{M}}(X, T) Y-R^{D^{M}}(Y, T) X, Z\right)^{v} U \\
& -G\left(X, \mathrm{P}_{M} Y\right)^{v}\left\{2 \mathrm{P}_{M} Z-\left(A \mathrm{P}_{M}+\mathrm{P}_{M} A\right) Z\right\}^{\uparrow},
\end{aligned}
$$

for any $X, Y, Z \in H(M)$. One should note (by $\theta(A Z)=0$, for any $Z \in H(M)$ ) that $u\left(a Z^{\uparrow}\right)=0$, i.e. $\left.u \circ a\right|_{H(N)}=0$. In particular $u(a \mathrm{P} X)=0$ for any $X \in H(N)$, where from

$$
\left\{\begin{array}{l}
\rho X=T_{U} X, \quad X \in H(N) \\
T_{U} H(N) \subseteq H(N)
\end{array}\right.
$$

\subsection{A vanishing theorem}

Let us prove Theorem 5. To this end, we consider a local orthonormal frame $\left\{X_{a}\right\}=\left\{X_{j}, J_{M} X_{j}\right\}$ of $H(M)$ (here $\left.G\left(X_{a}, X_{b}\right)=\delta_{a b}\right)$, so that $\left\{T, X_{a}\right\}$ is a (local) orthonormal frame of $T(M)$. The Ricci curvature of $D^{M}$ is

$$
\begin{aligned}
\operatorname{Ric}_{D^{M}}(Y, Z) & :=\operatorname{trace}\left\{V \mapsto R^{D^{M}}(V, Y) Z\right\} \\
& =\sum_{k=1}^{n} g\left(R^{D^{M}}\left(X_{k}, Y\right) Z, X_{k}\right) .
\end{aligned}
$$

On the other hand, $\left\{X_{a}^{\uparrow}\right\}=\left\{X_{j}^{\uparrow}, J_{M} X_{j}^{\uparrow}\right\}$ is an orthonormal frame of $H(N)$, hence $\left\{\xi, U, X_{a}^{\uparrow}\right\}$ is an orthonormal frame in $T(N)$. Then (by (5.13))

$$
\begin{aligned}
\operatorname{Ric}_{D}\left(Y^{\uparrow}, Z^{\uparrow}\right)= & \sum_{a=1}^{2 n-2} g\left(R^{D}\left(X_{a}^{\uparrow}, Y^{\uparrow}\right) Z^{\uparrow}, X_{a}^{\uparrow}\right) \\
= & \sum_{a=1}^{2 n-2}\left\{g\left(\left(R^{D^{M}}\left(X_{a}, Y\right) Z\right)^{\uparrow}, X_{a}^{\uparrow}\right)\right\} \\
& -\sum_{a=1}^{2 n-2}\left\{G\left(X_{a}, \mathrm{P}_{M} Y\right)^{v} G\left(\left[2 \mathrm{P}_{M} Z-\left(A \mathrm{P}_{M}+\mathrm{P}_{M} A\right) Z\right], X_{a}\right)^{v}\right\} \\
= & \sum_{a=1}^{2 n-2} G\left(R^{D^{M}}\left(X_{a}, Y\right) Z, X_{a}\right)^{v}-G\left(\left[2 \mathrm{P}_{M}-\left(A \mathrm{P}_{M}+\mathrm{P}_{M} A\right)\right] Z, \mathrm{P}_{M} Y\right)^{v},
\end{aligned}
$$


where from

$$
\operatorname{Ric}_{D}\left(Y^{\uparrow}, Z^{\uparrow}\right)=\operatorname{Ric}_{D^{M}}(Y, Z)^{v}-G\left(\left[2 \mathrm{P}_{M}-\left(A \mathrm{P}_{M}+\mathrm{P}_{M} A\right)\right] Z, \mathrm{P}_{M} Y\right)^{v} .
$$

Then, for $V=Y-i \mathrm{P}_{M} Y, W=Z-i \mathrm{P}_{M} Z \in T_{1,0}(M)$

$$
\operatorname{Ric}_{D}\left(V^{\uparrow}, \bar{W}^{\uparrow}\right)=\operatorname{Ric}_{D^{M}}(V, \bar{W})^{v}-G\left(\left[2 \mathrm{P}_{M}-\left(A \mathrm{P}_{M}+\mathrm{P}_{M} A\right)\right] \bar{W}, \mathrm{P}_{M} V\right)^{v} .
$$

On the other hand

$$
\begin{aligned}
G( & {\left.\left[2 \mathrm{P}_{M}-\left(A \mathrm{P}_{M}+\mathrm{P}_{M} A\right)\right] \bar{W}, \mathrm{P}_{M} V\right) } \\
& =G\left(2 \mathrm{P}_{M} \bar{W}, \mathrm{P}_{M} V\right)-G\left(A \mathrm{P}_{M} \bar{W}, \mathrm{P}_{M} V\right)-G\left(\mathrm{P}_{M} A \bar{W}, \mathrm{P}_{M} V\right) \\
& =G(2 \bar{W}, V)-2 G(A \bar{W}, V),
\end{aligned}
$$

so that

$$
G\left(\left[2 \mathrm{P}_{M}-\left(A \mathrm{P}_{M}+\mathrm{P}_{M} A\right)\right] \bar{W}, \mathrm{P}_{M} V\right)=2 G((\mathrm{I}-A) \bar{W}, V),
$$

leading to

$$
\operatorname{Ric}_{D}\left(V^{\uparrow}, \bar{W}^{\uparrow}\right)=\operatorname{Ric}_{D^{M}}(V, \bar{W})^{v}-2 G((\mathrm{I}-A) \bar{W}, V) .
$$

The identity (5.15) is the key ingredient in the proof of Theorems 5 and 6 . Let us recall that a complex valued $q$-form $\omega \in \Omega^{q}(M)$ is a $(0, q)$-form if

$$
\left.T\rfloor \omega=0, \quad T_{1,0}(M)\right\rfloor \omega=0 .
$$

The tangential Cauchy-Riemann operator (on $(0, q)$-forms, $q \geq 1$ ) is the differential operator

$$
\bar{\partial}_{M}: \Omega^{0, q}(M) \rightarrow \Omega^{0, q+1}(M)
$$

defined as follows. Let $\Lambda^{0, q}(M) \rightarrow M$ be the fibre bundle of the $(0, q)$-forms and set $\Omega^{0, q}(M):=\Gamma^{\infty}\left(\Lambda^{0, q}(M)\right)$. If $\omega \in \Omega^{0, q}(M)$ then $\bar{\partial}_{M} \omega$ is the unique $(0, q+1)$ form coinciding with $d \omega$ on $T_{0,1}(M) \otimes \cdots \otimes T_{0,1}(M)$ (q+1-terms). As well known

$$
C^{\infty}(M, \mathbf{C}) \stackrel{\bar{\partial}_{M}}{\rightarrow} \Omega^{0,1}(M) \stackrel{\bar{\partial}_{M}}{\rightarrow} \cdots \stackrel{\bar{\partial}_{M}}{\rightarrow} \Omega^{0, n-1}(M) \stackrel{\bar{\partial}_{M}}{\rightarrow} 0
$$

is a cochain complex (the tangential Cauchy-Riemann complex) and its cohomology

$$
H^{0, q}\left(M, \bar{\partial}_{M}\right):=H^{0, q}\left(\Omega^{0, \cdot}(M), \bar{\partial}_{M}\right)=\frac{\operatorname{Ker}\left\{\bar{\partial}_{M}: \Omega^{0, q}(M) \rightarrow \cdot\right\}}{\bar{\partial}_{M} \Omega^{0, q-1}(M)}
$$

is the so called Kohn-Rossi cohomology of $\left(M, T_{1,0}(M)\right)$. By a result of J. M. Lee (cf. [16]), if $M$ is compact and strictly pseudoconvex, and the (pseudohermitian) Ricci tensor of the Tanaka-Webster connection satisfies $\operatorname{Ric}_{D^{M}}(W, \bar{W})$ $\geq 0$, for any $W \in T_{1,0}(M)$, then $H^{0,1}\left(M, \bar{\partial}_{M}\right)=0$. This is clearly an analog of the well known Bochner theorem that the first Betti number of a compact Riemannian manifold of positive semi-definite Ricci curvature vanishes (yet 
$H^{0,1}\left(M, \bar{\partial}_{M}\right)$ is not a topological invariant). In the same spirit, by our assumption (1.5) and by (5.15) we may conclude as in Theorem $5 . \quad$ Q.e.d.

\subsection{C-R fibrations over a pseudo-Einstein manifold}

Let $\left(M, T_{1,0}(M)\right)$ be a nondegenerate $\mathrm{CR}$ manifold, of hypersurface type, of CR dimension $n$. Let $\theta$ be a contact form over $M$ and $D^{M}$ the TanakaWebster connection of $(M, \theta)$. If $\left\{T_{\alpha}\right\}$ is a local frame of $T_{1,0}(M)$ we set $R_{\alpha \bar{\beta}}=$ $\operatorname{Ric}_{D^{M}}\left(T_{\alpha}, T_{\bar{\beta}}\right)$ (the pseudohermitian Ricci tensor of $(M, \theta)$ ). Then $(M, \theta)$ is a pseudo-Einstein manifold if the pseudohermitian Ricci tensor is proportional to the Levi form (a CR analog to the Einstein condition in Riemannian geometry), i.e. $R_{\alpha \bar{\beta}}=\lambda g_{\alpha \bar{\beta}}$, for some $\lambda \in C^{\infty}(M)$. Here $g_{\alpha \bar{\beta}}=L_{\theta}\left(T_{\alpha}, T_{\bar{\beta}}\right)$. A posteriori $\lambda=\rho / n$, where $\rho=g^{\alpha \bar{\beta}} R_{\alpha \bar{\beta}}$ is the pseudohermitian scalar curvature of $(M, \theta)$. Examples are abundant. The sphere $S^{2 n+1}$ endowed with the standard contact structure (as hypersurface in $\mathbf{C}^{n+1}$ ) is a pseudo-Einstein manifold. The total space $U(M)$ (carrying the contact structure induced by the almost hermitian structure of $T(M)$ ) of the tangent sphere bundle over a real space-form $M$ of (costant) sectional curvature 1 is a pseudo-Einstein manifold (cf. E. Barletta \& S. Dragomir, [1]). If $f: M \rightarrow S^{2 n+1}$ is a pseudohermitian immersion with a flat normal TanakaWebster connection, of a compact strictly pseudoconvex $\mathrm{CR}$ manifold $M$ into a sphere, then $M$ admits a global pseudo-Einstein structure (cf. E. Barletta \& S. Dragomir, [2]).

As well known (cf. J. M. Lee, [16]) there are obstructions towards the existence of a globally defined pseudo-Einstein structure on a given nondegenerate CR manifold (for instance, if $\left(M, T_{1,0}(M)\right)$ admits a contact pseudo-Einstein structure then the first Chern class of the CR structure must vanish $\left(c_{1}\left(T_{1,0}(M)\right)\right.$ $=0)$ ). The Lee conjecture states that every compact strictly pseudoconvex CR manifold satisfying $c_{1}\left(T_{1,0}(M)\right)=0$ should actually possess a globally defined pseudo-Einstein structure (a CR analog of the Calabi problem). Positive answers to the Lee conjecture are known under additional assumptions (e.g. when $M$ admits a 1-parameter group of CR automorphisms transverse to $H(M)$, cf. J. M. Lee, [16], or when the contact vector field $T$ is regular in the sense of Palais, cf. S. Dragomir, [10]). Theorem 6 furnishes new examples of pseudo-Einstein manifolds, as base spaces of $\mathrm{C}-\mathrm{R}$ fibrations whose total space satisfies the assumption (1.6). Let us prove Theorem 6. By (5.15) for $V=T_{\alpha}$ and $W=T_{\beta}$

$$
\operatorname{Ric}_{D}\left(T_{\alpha}^{\uparrow}, T_{\bar{\beta}}^{\uparrow}\right)-2 G\left(T_{\alpha}, A T_{\bar{\beta}}\right)=R_{\alpha \bar{\beta}}-2 g_{\alpha \bar{\beta}}
$$

where $g_{\alpha \bar{\beta}}:=G\left(T_{\alpha}, T_{\bar{\beta}}\right)$. Assume that (1.6) holds. Then (by (3.5))

$$
R_{\alpha \bar{\beta}}=(\mu+2) g_{\alpha \bar{\beta}}
$$

so that $(M, \theta)$ is a pseudo-Einstein manifold of pseudohermitian scalar curvature $\rho=g^{\alpha \bar{\beta}} R_{\alpha \bar{\beta}}=(n-1)(\mu+2)$. Conversely, if (5.18) holds, then (by $T_{1,0}(N)=$ $T_{1,0}(M)^{\uparrow}$ and (5.17)) we obtain (1.6). Finally, if this is the case, by a result by J. M. Lee (cf. [16]) $c_{1}\left(T_{1,0}(M)\right)=0 . \quad$ Q.e.d. 


\section{REFERENCES}

[1] E. Barletta \& S. Dragomir, On the CR structure of the tangent sphere bundle, Le Matematiche, Catania, (2) L (1995), 237-249.

[2] E. Barletta \& S. Dragomir, Pseudohermitian immersions, pseudo-Einstein structures, and the Lee class of a CR manifold, Kodai Math. J., (1) 19 (1996), 62-86.

[ 3 ] A. Bejancu, CR submanifolds of a Kähler manifold, I-II, Proc. A.M.S., 69 (1978), 135-142; Trans. A.M.S., 250 (1979), 333-345.

[ 4 ] D. E. Blair, Contact manifolds in Riemannian geometry, Lecture Notes in Math., vol. 509, Springer-Verlag, Berlin-Heidelberg-New York, 1976.

[ 5 ] D. E. Blair \& B. Y. Chen, On CR submanifolds of Hermitian manifolds, Israel J. Math., 34 (1979), 353-369.

[6] A. Boggess, CR manifolds and the tangential Cauchy-Riemann complex, Studies in Advanced Math., CRC Press, Boca Raton-Ann Arbor-Boston-London, 1991.

[ 7 ] W. M. Воотнву \& H. C. WANG, On contact manifolds, Ann. of Math., (3) 68 (1958), $721-$ 734.

[8] S. Dragomir, Pseudohermitian immersions between strictly pseudoconvex CR manifolds, American J. Math., (1) 117 (1995), 169-202.

[9] S. Dragomir, A survey of pseudohermitian geometry, Suppl. ai Rendiconti del Circ. Matem. Palermo, Serie III, No. 49, 1997 [Proceedings of the Workshop on Diff. Geom. and Topology, Palermo (Italy), June 3-9, 1996].

[10] S. Dragomir, On a conjecture of J. M. Lee, Hokkaido Math. J., (1) XXIII (1994), 35-49.

[11] C. Fefferman, Monge-Ampère equations, the Bergman kernel and geometry of pseudoconvex domains, Ann. Math., (2) 103 (1976), 395-416; ibid., 104 (1976), 393-394.

[12] G. M. Henkin, The Hartogs-Bochner effect on CR manifolds, Soviet Math. Dokl., 29 (1984), 78-82.

[13] S. Ianuş, Sulle varietà di Cauchy-Riemann, Rend. dell'Accademia di Scienze Fisiche e Matematiche, Napoli, XXXIX (1972), 191-195.

[14] C. Laurent-ThiÉBaut, Résolution du $\bar{\partial}_{b}$ à support compact et phénomène de Hartogs-Bochner dans les variétés CR, Proceedings of Symposia in Pure Mathem., 52 (1991), 239-249.

[15] J. M. LeE, The Fefferman metric and pseudohermitian invariants, Trans. A.M.S., (1) 296 (1986), 411-429.

[16] J. M. LeE, Pseudo-Einstein structures on CR manifolds, American J. Math., 110 (1988), 157-178.

[17] R. Mizner, CR structures of codimension 2, J. Diff. Geometry, 30 (1989), 167-190.

[18] R. Mizner, Almost CR structures, $f$-structures, almost product structures and associated connections, Rocky Mountain J. Math., (4) 23 (1993), 1337-1359.

[19] B. O’NeILl, The fundamental equations of a submersion, Michigan Math. J., (4) 13 (1966), 459-469.

[20] R. S. Palais, A global formulation of the Lie theory of transformation groups, Memoirs of A.M.S., no. 22, 1957.

[21] N. TanaKa, A differenial geometric study on strongly psuedoconvex manifolds, Kinokunia Book Store Co., Ltd., Kyoto, 1975.

[22] G. Tomassins, Tracce delle funzioni olomorfe sulle varietà analitiche reali di una varietà complessa, Ann. Scuola Norm. Sup. Pisa, 20 (1966), 31-43.

[23] H. URakawa, Yang-Mills connections over compact strongly pseudoconvex CR manifolds, Math. Z., 216 (1994), 541-573.

[24] S. Webster, Pseudohermitian structures on a real hypersurface, J. Diff. Geometry, 13 (1978), $25-41$. 
[25] R. O. WeLLS, Holomorphic hulls and holomorphic convexity of differentiable submanifolds, Trans. A.M.S., 132 (1968), 245-262.

[26] R. O. Wells, Compact real submanifolds of a complex manifold with nondegenerate holomorphic tangent bundles, Math. Ann., 179 (1969), 123-129.

[27] K. Yano, On a structure defined by a tensor field of type $(1,1)$ satisfying $f^{3}+f=0$, Tensor, N.S., 14 (1963), 99-109.

[28] K. Yano \& M. Kon, Differential geometry of CR submanifolds, Geometriae Dedicata, 10 (1981), 369-391.

[29] K. Yano \& M. Kon, CR submanifolds of Kaehlerian and Sasakian Manifolds, Progress in Math., vol. 30, Ed. by J. Coates \& S. Helgason, Birkhäuser, Boston-Basel-Stuttgart, 1983.

POLITECNICO DI TORINO

DIMAT

Dipartimento di Matematica

Corso Duca Degli Abruzzi 24

TORINO, ITALIA

E-mail address: ciampax@tiscali.it 